Colimits, Stanley-Reisner algebras, and loop spaces

Panov, Taras and Ray, Nigel and Vogt, Rainer 2004

MIMS EPrint: 2006.338

Manchester Institute for Mathematical Sciences

School of Mathematics

The University of Manchester

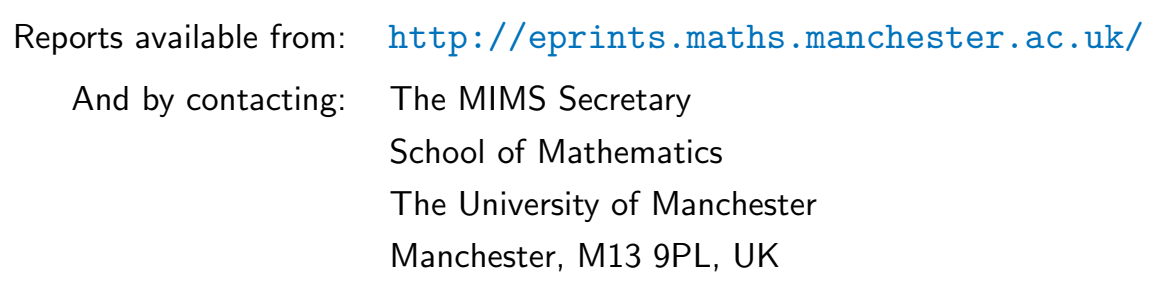

ISSN 1749-9097 


\title{
COLIMITS, STANLEY-REISNER ALGEBRAS, AND LOOP SPACES
}

\author{
TARAS PANOV, NIGEL RAY, AND RAINER VOGT
}

\begin{abstract}
We study diagrams associated with a finite simplicial complex $K$, in various algebraic and topological categories. We relate their colimits to familiar structures in algebra, combinatorics, geometry and topology. These include: right-angled Artin and Coxeter groups (and their complex analogues, which we call circulation groups); Stanley-Reisner algebras and coalgebras; Davis and Januszkiewicz's spaces $D J(K)$ associated with toric manifolds and their generalisations; and coordinate subspace arrangements. When $K$ is a flag complex, we extend well-known results on Artin and Coxeter groups by confirming that the relevant circulation group is homotopy equivalent to the space of loops $\Omega D J(K)$. We define homotopy colimits for diagrams of topological monoids and topological groups, and show they commute with the formation of classifying spaces in a suitably generalised sense. We deduce that the homotopy colimit of the appropriate diagram of topological groups is a model for $\Omega D J(K)$ for an arbitrary complex $K$, and that the natural projection onto the original colimit is a homotopy equivalence when $K$ is flag. In this case, the two models are compatible.
\end{abstract}

\section{INTRODUCTION}

In this work we study diagrams associated with a finite simplicial complex $K$, in various algebraic and topological categories. We are particularly interested in colimits and homotopy colimits of such diagrams.

We are motivated by Davis and Januszkiewicz's investigation [12] of toric manifolds, in which $K$ first arises as the boundary of the quotient polytope. In the course of their cohomological computations, Davis and Januszkiewicz construct real and complex versions of a space whose cohomology ring is isomorphic to the Stanley-Reisner algebra of $K$, over $\mathbb{Z} / 2$ and $\mathbb{Z}$ respectively. We denote the homotopy type of these spaces by $D J(K)$, and follow Buchstaber and Panov [7] by describing them as colimits of diagrams of classifying spaces. In this context, an exterior version arises naturally as an alternative. Suggestively, the cohomology algebras and homology coalgebras of the $D J(K)$ may be expressed as the limits and colimits of analogous diagrams in the corresponding algebraic category.

When colimits of similar diagrams are taken in a category of discrete groups, they yield right-angled Coxeter and Artin groups. These are more usually described by a complementary construction involving only the 1 -skeleton $K^{(1)}$ of $K$. Whenever $K$ is determined entirely by $K^{(1)}$ it is known as a flag complex, and results such as those of [12] and [22] may be interpreted as showing that the associated Coxeter and Artin groups are homotopy equivalent to the loop spaces $\Omega D J(K)$, in the real and exterior cases respectively. In other words, the groups are discrete models for the loop spaces. These observations raise the possibility of modelling

Key words and phrases. Colimit, flag complex, homotopy colimit, loop space, right-angled Artin group, right-angled Coxeter group, Stanley-Reisner ring, topological monoid.

The first author was supported by a Royal Society/NATO Postdoctoral Fellowship at the University of Manchester, and also by the Russian Foundation for Basic Research, grant number 01-01-00546. 
$\Omega D J(K)$ in the complex case, and for arbitrary $K$, by colimits of diagrams in a suitably defined category of topological monoids. Our primary aim is to carry out this programme.

Before we begin, we must therefore confirm that our categories are sufficiently cocomplete for the proposed colimits to exist. We show that this is indeed the case (as predicted by folklore), and explain how the complex version of $\Omega D J(K)$ is modelled by the colimit of a diagram of tori whenever $K$ is flag. We refer to the colimit as a circulation group, and consider it as the complex analogue of the corresponding right-angled Coxeter and Artin groups. Of course, it is also determined by $K^{(1)}$. On the other hand, there are simple examples of nonflag complexes for which the colimit groups cannot possibly model $\Omega D J(K)$ in any of the real, exterior, or complex cases. More subtle constructions are required.

Since we are engaged with homotopy theoretic properties of colimits, it is no great surprise that the appropriate model for arbitrary complexes $K$ is a homotopy colimit. Considerable care has to be taken in formulating the construction for topological monoids, but the outcome clarifies the status of the original colimits when $K$ is flag; flag complexes are precisely those for which the colimit and the homotopy colimit coincide. Our main result is therefore that $\Omega D J(K)$ is modelled by the homotopy colimit of the relevant diagram of topological groups, in all three cases and for arbitrary $K$. When $K$ is flag, the natural projection onto the original colimit is a homotopy equivalence, and is compatible with the two model maps. Our proof revolves around the fact that homotopy colimits commute with the classifying space functor, in a context which is considerably more general than is needed here.

For particular complexes $K$, our constructions have interesting implications for traditional homotopy theoretic invariants such as Whitehead products, Samelson products, and their higher analogues and iterates. We hope to deal with these issues in subsequent work [27].

We now summarise the contents of each section.

It is particularly convenient to use the language of enriched category theory, so we devote Section 2 to establishing the notation, conventions and results that we need. These include a brief discussion of simplicial objects and their realisations, and verification of the cocompleteness of our category of topological monoids in the enriched setting. Readers who are familiar with this material, or willing to refer back to Section 2 as necessary, may proceed directly to Section 3, where we introduce the relevant categories and diagrams associated with a simplicial complex $K$. They include algebraic and topological examples, amongst which are the exponential diagrams $G^{K}$; here $G$ denotes one of the cyclic groups $C_{2}$ and $C$, or the circle group $T$, in the real, exterior, and complex cases respectively.

We devote Section 4 to describing the limits and colimits of these diagrams. Some are identified with standard constructions such as the Stanley-Reisner algebra of $K$ and the Davis-Januszkiewicz spaces $D J(K)$, whereas the $G^{K}$ yield right-angled Coxeter and Artin groups, or circulation groups respectively. In Section 5 we study aspects of the diagrams involving associated fibrations and homotopy colimits. We note connections with coordinate subspace arrangements.

We introduce the model map $f_{K}: \operatorname{colim}^{\mathrm{TMG}} G^{K} \rightarrow \Omega D J(K)$ in Section 6, and determine the connectivity of its homotopy fibre in terms of combinatorial properties of $K$. The results confirm that $f_{K}$ is a homotopy equivalence whenever $K$ is flag, and quantify its failure for general $K$. In our final Section 7 we consider suitably well-behaved diagrams $D$ of topological monoids, and prove that the homotopy colimit of the induced diagram of classifying spaces is homotopy equivalent to the classifying space of the homotopy colimit of $D$, taken in the category of topological monoids. By application to the exponential diagrams $G^{K}$, we deduce 
that our generalised model map $h_{K}$ : hocolim ${ }^{\text {TMG }} G^{K} \rightarrow \Omega D J(K)$ is a homotopy equivalence for all complexes $K$. We note that the two models are compatible, and homotopy equivalent, when $K$ is flag.

We take the category TOP of $k$-spaces $X$ and continuous functions $f: X \rightarrow Y$ as our underlying topological framework, following [35]. Every function space $Y^{X}$ is endowed with the corresponding $k$-topology. Many of the spaces we consider have a distinguished basepoint $*$, and we write $\mathrm{TOP}_{+}$for the category of pairs $(X, *)$ and basepoint preserving maps; the forgetful functor $\mathrm{TOP}_{+} \rightarrow$ TOP is faithful. For any object $X$ of TOP, we may add a disjoint basepoint to obtain a based space $X_{+}$. The $k$-function space $(Y, *)^{(X, *)}$ has the trivial map $X \rightarrow *$ as basepoint. In some circumstances we need $(X, *)$ to be well-pointed, in the sense that the inclusion of the basepoint is a closed cofibration, and we emphasise this requirement as it arises.

Several other important categories are related to $\mathrm{TOP}_{+}$. These include $\mathrm{TMONH}$, consisting of associative topological monoids and homotopy homomorphisms [5] (essentially equivalent to Sugawara's strongly homotopy multiplicative maps [34]), and its subcategory TMON, in which the homorphisms are strict. Again, the forgetful functor $\mathrm{TMON} \rightarrow \mathrm{TOP}_{+}$is faithful. Limiting the objects to topological groups defines a further subcategory TGRP, which is full in TMON. In all three cases the identity element $e$ is the basepoint, and we may sometimes have to insist that objects are well-pointed. The Moore loop space $\Omega X$ is a typical object in TMONH for any pair $(X, *)$, and the canonical inclusion $M \rightarrow \Omega B M$ is a homotopy homomorphism for any well-pointed topological monoid $M$.

For each $m \geq 0$ we consider the small categories ID $(m)$, which consist of $m$ objects and their identity morphisms; in particular, we use the based versions $\operatorname{ID}_{\varnothing}(m)$, which result from adjoining an initial object $\varnothing$. Given a topological monoid $M$, the associated topological category $\mathrm{C}(M)$ consists of one object, and one morphism for each element of $M$. Segal's [32] classifying space $B \mathrm{C}(M)$ then coincides with the standard classifying space $B M$.

Given objects $X_{0}$ and $X_{n}$ of any category C, we denote the set of $n$-composable morphisms

$$
X_{0} \stackrel{f_{1}}{\longrightarrow} X_{1} \stackrel{f_{2}}{\longrightarrow} \cdots \stackrel{f_{n}}{\longrightarrow} X_{n}
$$

by $\mathrm{C}_{n}\left(X_{0}, X_{n}\right)$, for all $n \geq 0$. Thus $\mathrm{C}_{1}(X, Y)$ is the morphism set $\mathrm{C}(X, Y)$ for all objects $X$ and $Y$, and $\mathrm{C}_{0}(X, X)$ consists solely of the identity morphism on $X$.

In order to distinguish between them, we write $T$ for the multiplicative topological group of unimodular complex numbers, and $S^{1}$ for the circle. Similarly, we discriminate between the cyclic group $C_{2}$ and the ring of residue classes $\mathbb{Z} / 2$, and between the infinite cyclic group $C$ and the ring of integers $\mathbb{Z}$.

The first and second authors benefitted greatly from illuminating discussions with Bill Dwyer at the International Conference on Algebraic Topology held on the Island of Skye during June 2001. They are particularly grateful to the organisers for providing the opportunity to work in such magnificent surroundings.

\section{Categorical Prerequisites}

We refer to the books of Kelly [21] and Borceux [3] for notation and terminology associated with the theory of enriched categories, and to Barr and Wells [1] for background on the theory of monads (otherwise known as triples). For more specific results, we cite [14] and [18]. Unless otherwise stated, we assume that all our categories are enriched in one of the topological senses 
described below, and that functors are continuous. In many cases the morphism sets are finite, and therefore invested with the discrete topology.

Given an arbitrary category $\mathrm{R}$, we refer to a covariant functor $D: \mathrm{A} \rightarrow \mathrm{R}$ as an A-diagram in $\mathrm{R}$, for any small category A. Such diagrams are the objects of a category $[A, R]$, whose morphisms are natural transformations of functors. We may interpret any object $X$ of $\mathrm{R}$ as a constant diagram, which maps each object of a to $X$ and every morphism to the identity.

Examples 2.1. Let $\Delta$ be the category whose objects are the ordinals $(n)=\{0,1, \ldots, n\}$, where $n \geq 0$, and whose morphisms are the nondecreasing functions; then $\Delta^{o p}$ - and $\Delta$-diagrams are simplicial and cosimplicial objects of A respectively. In particular, $\Delta: \Delta \rightarrow$ TOP is the cosimplicial space which assigns the standard $n$-simplex $\Delta(n)$ to each object $(n)$. Its pointed analogue $\Delta_{+}$is given by $\Delta_{+}(n)=\Delta(n)_{+}$.

If $M$ is a topological monoid, then $\mathrm{C}(M)$ - and $\mathrm{C}(M)^{\text {op }}$-diagrams in $\mathrm{TOP}$ are left and right $M$-spaces respectively.

We recall that $(\mathrm{S}, \square, \Phi)$ is a symmetric monoidal category if the bifunctor $\square: \mathrm{S} \times \mathrm{S} \rightarrow \mathrm{S}$ is coherently associative and commutative, and $\Phi$ is a coherent unit object. Such an $\mathrm{S}$ is closed if there is a bifunctor $\mathrm{S} \times \mathrm{S}^{o p} \rightarrow \mathrm{S}$, denoted by $(Z, Y) \mapsto[Y, Z]$, which satisfies the adjunction

$$
\mathrm{s}(X \square Y, Z) \cong \mathrm{s}(X,[Y, Z])
$$

for all objects $X, Y$, and $Z$ of s. A category $\mathrm{R}$ is s-enriched when its morphism sets are identified with objects of $\mathrm{s}$, and composition factors naturally through $\square$. A closed symmetric monoidal category is canonically self-enriched, by identifying $\mathrm{s}(X, Y)$ with $[X, Y]$. Henceforth, s denotes such a category.

Example 2.2. Any small $\mathrm{S}-$ enriched category A determines a diagram $A: \mathrm{A} \times \mathrm{A}^{o p} \rightarrow \mathrm{S}$, whose value at $(a, b)$ is the morphism object $\mathrm{A}(b, a)$.

An S-functor $\mathrm{Q} \rightarrow \mathrm{R}$ of S-enriched categories acts on morphism sets as a morphism of $\mathrm{S}$. The category $[Q, R]$ of such functors has morphisms consisting of natural transformations, and is also S-enriched. The s-functors $F: \mathrm{Q} \rightarrow \mathrm{R}$ and $U: \mathrm{R} \rightarrow \mathrm{Q}$ are s-adjoint if there is a natural isomorphism

$$
\mathrm{R}(F(X), Y) \cong \mathrm{Q}(X, U(Y))
$$

in $\mathrm{s}$, for all objects $X$ of $\mathrm{Q}$ and $Y$ of R.

Examples 2.3. The categories TOP and $\mathrm{TOP}_{+}$are symmetric monoidal under cartesian product $\times$ and smash product $\wedge$ respectively, with unit objects the one-point space $*$ and the zerosphere $*_{+}$. Both are closed, and therefore self-enriched, by identifying $[X, Y]$ with $Y^{X}$ and $(Y, *)^{(X, *)}$ respectively.

Since $(Y, *)^{(X, *)}$ inherits the subspace topology from $Y^{X}$, the induced Top-enrichment of $\mathrm{TOP}_{+}$is compatible with its self-enrichment. Both TMON and TGRP are TOP + -enriched by restriction.

In certain situations it is helpful to reserve the notation $\mathrm{T}$ for either or both of the selfenriched categories TOP and TOP + . Similarly, we reserve TMG for either or both of the $\mathrm{TOP}_{+}$-enriched categories TMON and TGRP.

It is well known that TOP and $\mathrm{TOP}_{+}$are complete and cocomplete, in the standard sense that every small diagram has a limit and colimit. Completeness is equivalent to the existence of products and equalizers, and cocompleteness to the existence of coproducts and coequalizers. Both TOP and TOP + actually admit indexed limits and indexed colimits [21], involving 
topologically parametrized diagrams in the enriched setting; in other words, $\mathrm{T}$ is $\mathrm{T}$-complete and T-cocomplete. A summary of the details for TOP can be found in [26].

Amongst indexed limits and colimits, the enriched analogues of products and coproducts are particularly important.

Definitions 2.4. An S-enriched category $\mathrm{R}$ is tensored and cotensored over $\mathrm{S}$ if there exist bifunctors $\mathrm{R} \times \mathrm{S} \rightarrow \mathrm{R}$ and $\mathrm{R} \times \mathrm{S}^{o p} \rightarrow \mathrm{R}$ respectively, denoted by

$$
(X, Y) \longmapsto X \otimes Y \quad \text { and } \quad(X, Y) \longmapsto X^{Y},
$$

together with natural isomorphisms

$$
\mathrm{R}(X \otimes Y, Z) \cong \mathrm{s}(Y, \mathrm{R}(X, Z)) \cong \mathrm{R}\left(X, Z^{Y}\right)
$$

in $\mathrm{s}$, for all objects $X, Z$ of $\mathrm{R}$ and $Y$ of $\mathrm{s}$.

For any such $\mathrm{R}$, there are therefore natural isomorphisms

$$
X \otimes \Phi \cong X \cong X^{\Phi} \quad \text { and } \quad X \otimes(Y \square W) \cong(X \otimes Y) \otimes W .
$$

Every $\mathrm{S}$ is tensored over itself by $\square$, and cotensored by [, ].

Examples 2.7. The categories $\mathrm{T}$ are tensored and cotensored over themselves; so $X \otimes Y$ and $X^{Y}$ are given by $X \times Y$ and $X^{Y}$ in $\mathrm{TOP}$, and by $X \wedge Y$ and $(Y, *)^{(X, *)}$ in $\mathrm{TOP}_{+}$.

The rôle of tensors and cotensors is clarified by the following results of Kelly [21, (3.69)(3.73)]. Here and henceforth, we take $\mathrm{s}$ to be complete and cocomplete in the standard sense.

Theorem 2.8. An S-enriched category is S-complete if and only if it is complete, and cotensored over $\mathrm{S}$; it is $\mathrm{S}$-cocomplete if and only if it is cocomplete, and tensored over $\mathrm{S}$.

Theorem 2.8 asserts that standard limits and colimits may themselves be enriched in the presence of tensors and cotensors, since they are special cases of indexed limits and colimits. Given an A-diagram $D$ in $\mathrm{R}$, where $\mathrm{A}$ is also S-enriched, we deduce that the natural bijections

$$
\mathrm{R}(X, \lim D) \longleftrightarrow[\mathrm{A}, \mathrm{R}](X, D) \quad \text { and } \quad \mathrm{R}(\operatorname{colim} D, Y) \longleftrightarrow[\mathrm{A}, \mathrm{R}](D, Y)
$$

are isomorphisms in s, for any objects $X$ and $Y$ of R.

It is convenient to formulate several properties of TMON and TGRP by observing that both categories are $\mathrm{TOP}_{+}$-complete and -cocomplete. We appeal to the monad associated with the forgetful functor $U: \mathrm{TMG} \rightarrow \mathrm{TOP}_{+}$; in both cases it has a left $\mathrm{TOP}_{+}$-adjoint, given by the free monoid or free group functor $F$. The composition $U \cdot F$ defines a TOP + -monad $L: \mathrm{TOP}_{+} \rightarrow \mathrm{TOP}_{+}$, whose category $\mathrm{TOP}_{+}^{L}$ of algebras is precisely TMG. We write $V$ for the forgetful functor TGRP $\rightarrow$ TMON, whose left TOP + -adjoint is the universal group functor.

Proposition 2.10. The categories TMON and TGRP are $\mathrm{TOP}_{+}$-complete and-cocomplete; moreover, $V$ preserves indexed colimits.

Proof. We consider the forgetful functor $\mathrm{TOP}_{+}^{L} \rightarrow \mathrm{TOP}_{+}$, noting that $\mathrm{TOP}_{+}$is $\mathrm{TOP}_{+}-$complete by Theorem 2.8 .

Part (i) of [14, VII, Proposition 2.10] asserts that the forgetful functor creates all indexed limits, confirming that TMG is $\mathrm{TOP}_{+}$-complete. Part (ii) asserts that $\mathrm{TOP}_{+}^{L}$ is $\mathrm{TOP}_{+}$-cocomplete if $L$ preserves reflexive coequalizers, which need only be verified for $U$ because $F$ preserves colimits. The result follows for an arbitrary reflexive pair $(f, g)$ in TMG by using the right inverse to show that the coequalizer of $(U(f), U(g))$ in TOP + is itself in the image of $U$, and lifts to the coequalizer of $(f, g)$. 
Finally, $V$ preserves indexed colimits because it is left adjoint to a TOP + -functor TMON $\rightarrow$ TGRP, which associates to each topological monoid its subgroup of invertible elements.

In view of Proposition 2.10 we may form colimits of diagrams in TMG by applying colim ${ }^{\text {TMON }}$, even when the diagram consists entirely of topological groups. Pioneering results on the completeness and cocompleteness of categories of topological monoids and topological groups may be found in [6].

Our main deduction from Proposition 2.10 is that TMON and TGRP are tensored over $\mathrm{TOP}_{+}$. By studying the isomorphisms (2.5), we may construct the tensors explicitly; they are described as pushouts in [30, 2.2].

Construction 2.11. For any objects $M$ of TMON and $Y$ of $\mathrm{TOP}_{+}$, the tensored monoid $M \circledast Y$ is the quotient of the free topological monoid on $U(M) \wedge Y$ by the relations

$$
(m, y)\left(m^{\prime}, y\right)=\left(m m^{\prime}, y\right) \quad \text { for all } m, m^{\prime} \in M \text { and } y \in Y .
$$

For any object $G$ of TGRP, the tensored group $G \circledast Y$ is the topological group $V(G) \circledast Y$.

The cotensored monoid $M^{Y}$ and cotensored group $G^{Y}$ are the function spaces $\operatorname{TOP}_{+}(Y, M)$ and $\operatorname{TOP}_{+}(Y, G)$ respectively, under pointwise multiplication.

Given a category $\mathrm{R}$ which is tensored and cotensored over $\mathrm{S}$, we may now describe several categorical constructions. They are straightforward variations on [18, 2.3], and initially involve three diagrams. The first is $D: \mathrm{B}^{o p} \rightarrow \mathrm{R}$, the second $E: \mathrm{B} \rightarrow \mathrm{s}$, and the third $F: \mathrm{B} \rightarrow \mathrm{R}$.

Definitions 2.12. The tensor product $D \otimes_{\mathrm{B}} E$ is the coequalizer of

$$
\coprod_{g: b_{0} \rightarrow b_{1}} D\left(b_{1}\right) \otimes E\left(b_{0}\right) \stackrel{\alpha}{\underset{\beta}{\rightleftarrows}} \coprod_{b} D(b) \otimes E(b)
$$

in $\mathrm{R}$, where $g$ ranges over the morphisms of $\mathrm{B}$, and $\left.\alpha\right|_{g}=D(g) \otimes 1$ and $\left.\beta\right|_{g}=1 \otimes E(g)$. The homset $\operatorname{Hom}_{\mathrm{B}}(E, F)$ is the equalizer of

$$
\prod_{b} F(b)^{E(b)} \underset{\beta}{\stackrel{\alpha}{\rightleftarrows}} \prod_{g: b_{0} \rightarrow b_{1}} F\left(b_{1}\right)^{E\left(b_{0}\right)}
$$

in $\mathrm{R}$, where $\alpha=\prod_{g} \cdot E(g)$ and $\beta=\prod_{g} F(g) \cdot$

We may interpret the elements of $\operatorname{Hom}_{\mathrm{B}}(E, F)$ as mappings from the diagram $E$ to the diagram $F$, using the cotensor pairing.

Examples 2.13. Consider the case $\mathrm{R}=\mathrm{S}=\mathrm{TOP}$ or $\mathrm{TOP}_{+}$, with $\mathrm{B}=\Delta$. Given simplicial spaces $X_{\bullet}: \Delta^{o p} \rightarrow$ TOP and $Y_{\bullet}: \Delta^{o p} \rightarrow \mathrm{TOP}_{+}$, the tensor products

$$
\left|X_{\bullet}\right|=X_{\bullet} \times_{\Delta} \Delta \quad \text { and } \quad\left|Y_{\bullet}\right|=Y_{\bullet} \wedge_{\Delta} \Delta_{+}
$$

represent their topological realisation [24] in $\mathrm{TOP}$ and $\mathrm{TOP}_{+}$respectively. If we choose $\mathrm{R}=\mathrm{TMG}$ and $\mathrm{S}=\mathrm{TOP}_{+}$, a simplicial object $M_{\bullet}: \Delta^{o p} \rightarrow \mathrm{TMG}$ has internal and topological realisations

$$
\left|M_{\bullet}\right|_{\mathrm{TMG}}=M_{\bullet} \circledast \Delta_{+} \quad \text { and } \quad\left|M_{\bullet}\right|=U(M) \bullet \wedge_{\Delta} \Delta_{+}
$$

in $\mathrm{TMG}$ and $\mathrm{TOP}_{+}$respectively. Since || preserves products, $\left|M_{\bullet}\right|$ actually lies in TMG.

If $\mathrm{R}=\mathrm{s}$, then $D \otimes_{\mathrm{B}} \Phi$ is colim $D$, where $\Phi$ is the trivial $\mathrm{B}$-diagram. Also, $\operatorname{Hom}_{\mathrm{B}}(E, F)$ is the morphism set $[\mathrm{B}, \mathrm{R}](E, F)$, consisting of the natural transformations $E \rightarrow F$.

For $Y_{\bullet}$ in Examples 2.13, its TOP- and TOP+-realisations are homeomorphic because basepoints of the $Y_{n}$ represent degenerate simplices for $n>0$. We identify $\left|M_{\bullet}\right|_{\text {TMG }}$ with $\left|M_{\bullet}\right|$ in Section 7. 
We need certain generalisations of Definitions 2.12, in which analogies with homological algebra become apparent. We extend the first and second diagrams to $D: \mathrm{A} \times \mathrm{B}^{o p} \rightarrow \mathrm{R}$ and $E: \mathrm{B} \times \mathrm{C}^{o p} \rightarrow \mathrm{S}$, and replace the third by $F: \mathrm{C} \times \mathrm{D}^{o p} \rightarrow \mathrm{S}$ or $G: \mathrm{A} \times \mathrm{C}^{o p} \rightarrow \mathrm{R}$. Then $D \otimes_{\mathrm{B}} E$ becomes an $\left(\mathrm{A} \times \mathrm{C}^{o p}\right)$-diagram in $\mathrm{R}$, and $\operatorname{Hom}_{\mathrm{C}^{o p}}(E, G)$ becomes an $\left(\mathrm{A} \times \mathrm{B}^{o p}\right)$-diagram in $\mathrm{R}$. The extended diagrams reduce to the originals by judicious substitution, such as $\mathrm{A}=\mathrm{C}=\mathrm{ID}$ in $D$ and $E$.

Example 2.14. Consider the case $\mathrm{R}=\mathrm{S}=\mathrm{TOP}_{+}$, with $\mathrm{A}=\mathrm{C}=\mathrm{ID}$ and $\mathrm{B}=\Delta$. Given $E=$ $\Delta_{+}$as before, and $G$ a constant diagram $Z: \mathrm{ID} \rightarrow \mathrm{TOP}_{+}$, then $\operatorname{Hom}_{\mathrm{C} o p}(E, G)$ coincides with the total singular complex $\operatorname{Sin}(Z)$ as an object of $\left[\Delta^{o p}, \mathrm{TOP}_{+}\right]$. If $\mathrm{R}=\mathrm{TMG}$ and $N: \mathrm{ID} \rightarrow \mathrm{TMG}$ is a constant diagram, then $\operatorname{Sin}(N)$ is an object of $\left[\Delta^{o p}, \mathrm{TMG}\right]$.

Important properties of tensor products are described by the natural equivalences

$$
D \otimes_{\mathrm{B}} B \cong D \quad \text { and } \quad\left(D \otimes_{\mathrm{B}} E\right) \otimes_{\mathrm{C}} F \cong D \otimes_{\mathrm{B}}\left(E \otimes_{\mathrm{C}} F\right)
$$

of $\left(\mathrm{A} \times \mathrm{B}^{o p}\right)$ - and $\left(\mathrm{A} \times \mathrm{D}^{o p}\right)$-diagrams respectively, in $\mathrm{R}$. The first equivalence applies Example 2.2 with $\mathrm{A}=\mathrm{B}$, and the second uses the isomorphism of (2.6). The adjoint relationship between $\otimes$ and Hom is expressed by the equivalences

$$
\left[\mathrm{A} \times \mathrm{C}^{o p}, \mathrm{R}\right]\left(D \otimes_{\mathrm{B}} E, G\right) \cong\left[\mathrm{B} \times \mathrm{C}^{o p}, \mathrm{~S}\right](E,[\mathrm{~A}, \mathrm{R}](D, G)) \cong\left[\mathrm{A} \times \mathrm{B}^{o p}, \mathrm{R}\right]\left(D, \operatorname{Hom}_{\mathrm{C}} o p(E, G)\right),
$$

which extend the tensor-cotensor relations (2.5), and are a consequence of the constructions.

Examples 2.17. Consider the data of Example 2.14, and suppose that $D$ is a simplicial pointed space $Y_{\bullet}: \Delta^{o p} \rightarrow \mathrm{TOP}_{+}$. Then the adjoint relation (2.16) provides a homeomorphism

$$
\mathrm{TOP}_{+}\left(\left|Y_{\bullet}\right|, Z\right) \cong\left[\Delta^{o p}, \mathrm{TOP}_{+}\right]\left(Y_{\bullet}, \operatorname{Sin}(Z)\right) .
$$

If $\mathrm{R}=\mathrm{TMG}$ and $\mathrm{S}=\mathrm{TOP}_{+}$, and $M_{\bullet}$ is a simplicial object in $\mathrm{TMG}$, we obtain a homeomorphism

$$
\operatorname{TMG}\left(\left|M_{\bullet}\right|_{\mathrm{TMG}}, N\right) \cong\left[\Delta^{o p}, \mathrm{TMG}\right]\left(M_{\bullet}, \operatorname{Sin}(N)\right)
$$

for any object $N$ of TMG.

If $\mathrm{R}=\mathrm{S}$ and $E=\Phi$, the relations (2.16) reduce to the second isomorphism (2.9).

The first two examples extend the classic adjoint relationship between | | and Sin.

We now assume $\mathrm{R}=\mathrm{S}=$ TOP. We let $D$ be an $\left(\mathrm{A} \times \mathrm{B}^{o p}\right)$-diagram as above, and define $B_{\bullet}(*, \mathrm{~A}, D)$ to be a degenerate form of the 2-sided bar construction. It is a $\mathrm{B}^{o p}$-diagram of simplicial spaces, given as a $\mathrm{B}^{o p} \times \Delta^{o p}$-diagram in TOP by

$$
(b,(n)) \longmapsto \bigsqcup_{a_{0}, a_{n}} D\left(b, a_{0}\right) \times \mathrm{A}_{n}\left(a_{0}, a_{n}\right)
$$

for each object $b$ of $\mathrm{B}$; the face and degeneracy maps are described as in [18] by composition (or evaluation) of morphisms and by the insertion of identities respectively. The topological realisation $B(*, \mathrm{~A}, D)$ is a $\mathrm{B}^{o p}$-diagram in TOP. This definitions ensure the existence of natural equivalences

$$
B_{\bullet}(*, \mathrm{~A}, D) \times_{\mathrm{B}} E \cong B_{\bullet}\left(*, \mathrm{~A}, D \times_{\mathrm{B}} E\right) \quad \text { and } \quad B(*, \mathrm{~A}, D) \times_{\mathrm{B}} E \cong B\left(*, \mathrm{~A}, D \times_{\mathrm{B}} E\right)
$$

of $\mathrm{C}^{o p}$-diagrams in $\left[\Delta^{o p}, \mathrm{TOP}\right]$ and TOP respectively.

Examples 2.20. If $\mathrm{B}=\mathrm{ID}$, the homotopy colimit [4] of a diagram $D: \mathrm{A} \rightarrow \mathrm{TOP}$ is given by

$$
\text { hocolim } D=B(*, \mathrm{~A}, D) \text {, }
$$

as explained in [18]; using (2.15) and (2.19), it is homeomorphic to both of

$$
B(*, \mathrm{~A}, A) \times_{\mathrm{A}} D \cong D \times_{\mathrm{A}^{o p}} B(*, \mathrm{~A}, A) .
$$


In particular, $B_{\bullet}(*, \mathrm{~A}, *)$ is the nerve [32] $B_{\bullet} \mathrm{A}$ of $\mathrm{A}$, whose realisation is the classifying space $B$ A of A. The natural projection hocolim $D \rightarrow \operatorname{colim} D$ is given by the map

$$
D \times_{\mathrm{A}^{o p}} B(*, \mathrm{~A}, A) \longrightarrow D \times_{\mathrm{A}^{o p}} *,
$$

induced by collapsing $B(*, \mathrm{~A}, A)$ onto $*$.

If $\mathrm{A}=\mathrm{C}(M)$, where $M$ is an arbitrary topological monoid, then $D$ is a left $M$-space and $B(*, \mathrm{C}(M), C(M))$ is a universal contractible right $M$-space EM [13]. So

$$
\text { hocolim } D=B(*, \mathrm{C}(M), C(M)) \times_{\mathrm{C}(M)} D
$$

is a model for the Borel construction $E M \times_{M} D$.

\section{Basic Constructions}

We choose a universal set $V$ of vertices $v_{1}, \ldots, v_{m}$, and let $K$ denote a simplicial complex with faces $\sigma \subseteq V$. The integer $|\sigma|-1$ is the dimension of $\sigma$, and the greatest such integer is the dimension of $K$. For each $1 \leq j \leq m$, the faces of dimension less than or equal to $j$ form a subcomplex $K^{(j)}$, known as the $j$-skeleton of $K$; in particular, the 1 -skeleton $K^{(1)}$ is a graph. We abuse notation by writing $V$ for the zero-skeleton of $K$, more properly described as $\left\{\left\{v_{j}\right\}: 1 \leq j \leq m\right\}$. At the other extreme we have the $(m-1)$-simplex, which is the complex containing all subsets of $V$; it is denoted by $2^{V}$ in the abstract setting and by $\Delta(V)$ when emphasising its geometrical realisation. Any simplicial complex $K$ therefore lies in a chain

$$
V \longrightarrow K \longrightarrow 2^{V}
$$

of subcomplexes. Every face $\sigma$ may also be interpreted as a subcomplex of $K$, and so masquerades as a $(|\sigma|-1)$-simplex.

A subset $W \subseteq V$ is a missing face of $K$ if every proper subset lies in $K$, yet $W$ itself does not; its dimension is $|W|-1$. We refer to $K$ as a flag complex, or write that $K$ is flag, when every missing face has two vertices. The boundary of a planar $m$-gon is therefore flag whenever $m \geq 4$, as is the barycentric subdivision $K^{\prime}$ of an arbitrary complex $K$. The flagification $F l(K)$ of $K$ is the minimal flag complex containing $K$ as a subcomplex, and is obtained from $K$ by adjoining every missing face containing three or more vertices.

Example 3.2. For any $n>2$, the simplest non-flag complex on $n$ vertices is the boundary of an $(n-1)$-simplex, denoted by $\partial(n)$; then $F l(\partial(n))$ is $\Delta(n-1)$ itself.

Given a subcomplex $K \subseteq L$ on vertices $V$, it is useful to define $W \subseteq V$ as a missing face of the pair $(L, K)$ whenever $W$ fails to lie in $K$, yet every proper subset lies in $L$.

Every finite simplicial complex $K$ gives rise to a finite category $\operatorname{CAT}(K)$, whose objects are the faces $\sigma$ and morphisms the inclusions $\sigma \subseteq \tau$. The empty face $\varnothing$ is an initial object. For any subcomplex $K \subseteq L$, the category $\operatorname{CAT}(K)$ is a full subcategory of $\operatorname{CAT}(L)$; in particular, (3.1) determines a chain of subcategories

$$
\mathrm{ID}_{\varnothing}(m) \longrightarrow \mathrm{CAT}(K) \longrightarrow \mathrm{CAT}\left(2^{V}\right) .
$$

For each face $\sigma$, we define the undercategory $\sigma \downarrow \operatorname{CAT}(K)$ by restricting attention to those objects $\tau$ for which $\sigma \subseteq \tau$; thus $\sigma$ is an initial object. Insisting that the inclusion $\sigma \subset \tau$ be strict yields the subcategory $\sigma \Downarrow \operatorname{CAT}(K)$, obtained by deleting $\sigma$. The overcategories $\operatorname{CAT}(K) \downarrow \sigma$ and $\operatorname{CAT}(K) \Downarrow \sigma$ are defined likewise.

A complex $K$ also determines a simplicial set $S(K)$, whose nondegenerate simplices are exactly the faces of $K[24]$. So the nerve $B \cdot \mathrm{CAT}(K)$ coincides with the simplicial set $S\left(\operatorname{Con}\left(K^{\prime}\right)\right)$, 
where $\operatorname{Con}\left(K^{\prime}\right)$ denotes the cone on the barycentric subdivision of $K$, and the cone point corresponds to $\varnothing$. More generally, $B(\sigma \downarrow \operatorname{CAT}(K))$ is the cone on $B(\sigma \Downarrow \operatorname{CAT}(K))$.

Examples 3.4. If $K=V$, then $B \operatorname{ID} \varnothing(m)$ is the cone on $m$ disjoint points. If $K=2^{V}$, then $B \operatorname{CAT}\left(2^{V}\right)$ is homeomorphic to the unit cube $I^{V} \subset \mathbb{R}^{V}$, and defines its canonical simplicial subdivision; the homeomorphism maps each vertex $\sigma \subseteq V$ to its characteristic function $\chi_{\sigma}$, and extends by linearity. If $K$ is the subcomplex $\partial(m)$, then $\operatorname{BCAT}(\partial(m))$ is obtained from the boundary $\partial I^{m}$ by deleting all faces which contain the maximal vertex $(1, \ldots, 1)$.

The undercategories define a $\operatorname{CAT}(K)^{o p}$-diagram $\downarrow \operatorname{CAT}(K)$ in the category of small categories. It takes the value $\sigma \downarrow \operatorname{CAT}(K)$ on each face $\sigma$, and the inclusion functor $\tau \downarrow \operatorname{CAT}(K) \subseteq$ $\sigma \downarrow \operatorname{CAT}(K)$ on each reverse inclusion $\tau \supseteq \sigma$. The formation of classifying spaces yields a $\operatorname{CAT}(K)^{o p}$-diagram $B(\downarrow \mathrm{CAT}(K))$ in $\mathrm{TOP}_{+}$, which consists of cones and their inclusions. It takes the value $B(\sigma \downarrow \operatorname{CAT}(K))$ on $\sigma$ and $B(\tau \downarrow \operatorname{CAT}(K)) \subseteq B(\sigma \downarrow \operatorname{CAT}(K)$ on $\tau \supseteq \sigma$, and its colimit is the final space $B \mathrm{CAT}(K)$. Following [18], we note the isomorphism

$$
B(\downarrow \mathrm{CAT}(K)) \cong B(*, \mathrm{CAT}(K), C A T(K))
$$

of $\operatorname{CAT}(K)^{o p}$-diagrams in $\mathrm{TOP}_{+}$.

We refer to the cones $B(\sigma \downarrow \mathrm{CAT}(K))$ as faces of $B \mathrm{CAT}(K)$, amongst which we distinguish the facets $B(v \downarrow \operatorname{CAT}(K))$, defined by the vertices $v$. The facets determine the faces, according to the expression

$$
B(\sigma \downarrow \operatorname{CAT}(K))=\bigcap_{v \in \sigma} B(v \downarrow \operatorname{CAT}(K))
$$

for each $\sigma \in K$, and form a panel structure on $\operatorname{BCAT}(K)$ as described by Davis [11]. This terminology is motivated by our next example, which lies at the heart of recent developments in the theory of toric manifolds.

Example 3.6. The boundary of a simplicial polytope $P$ is a simplicial complex $K_{P}$, with faces $\sigma$. The polar $P^{*}$ of $P$ is a simple polytope of the same dimension, whose faces $F_{\sigma}$ are dual to those of $P$ (it is convenient to consider $F_{\varnothing}$ as $P^{*}$ itself). There is a homeomorphism $\operatorname{BCAT}\left(K_{P}\right) \rightarrow P^{*}$, which maps each vertex $\sigma$ to the barycentre of $F_{\sigma}$, and transforms each face $B(\sigma \downarrow \mathrm{CAT}(K))$ homeomorphically onto $F_{\sigma}$.

Classifying the categories and functors of (3.3) yields the chain of subspaces

$$
\operatorname{Con}(V) \longrightarrow \operatorname{BCAT}(K) \longrightarrow I^{m} \text {. }
$$

So $B \operatorname{CAT}(K)$ contains the unit axes, and is a subcomplex of $I^{m}$. It is therefore endowed with the induced cubical structure, as are all subspaces $B(\sigma \downarrow \mathrm{CAT}(K))$. In particular, the simple polytope $P^{*}$ of Example 3.6 admits a natural cubical decomposition.

In our algebraic context, we utilise the category GRP of discrete groups and homomorphisms. Many constructions in GRP may be obtained by restriction from those we describe in TMON, and we leave readers to provide the details. In particular, GRP is a full subcategory of $\mathrm{TMG}$, and is $\mathrm{TOP}_{+}$-complete and -cocomplete.

Given a commutative ring $Q$ (usually the integers, or their reduction mod 2), we consider the category $Q^{\mathrm{MOD}}$ of left $Q$-modules and $Q$-linear maps, which is symmetric monoidal with respect to the tensor product $\otimes_{Q}$ and closed under $(Z, Y) \mapsto{ }_{Q} \operatorname{MOD}(Y, Z)$. We usually work in the related category $\mathrm{G}_{Q} \mathrm{MOD}$ of connected graded modules of finite type, or more particularly in the categories $\mathrm{G}_{Q} \mathrm{CALG}$ and $\mathrm{G}_{Q} \mathrm{COCOA}$, which are dual; the former consists of augmented commutative $Q$-algebras and their homomorphisms, and the latter of supplemented cocommutative $Q$-coalgebras and their coalgebra maps. 
As an object of ${ }_{Q} \mathrm{MOD}$, the polynomial algebra $Q[V]$ on $V$ has a basis of monomials $v_{W}=$ $\prod_{W} v_{j}$, for each multiset $W$ on $V$. Henceforth, we assign a common dimension $d\left(v_{j}\right)>0$ to the vertices $v_{j}$ for all $1 \leq j \leq m$, and interpret $Q[V]$ as an object of $\mathrm{G}_{Q}$ CALG; products are invested with appropriate signs if $d\left(v_{j}\right)$ is odd and $2 Q \neq 0$. Then the quotient map

$$
Q[V] \longrightarrow Q[V] /\left(v_{\lambda}: \lambda \notin K\right)
$$

is a morphism in $\mathrm{G}_{Q} \mathrm{CALG}$, whose target is known as the graded Stanley-Reisner Q-algebra of the simplicial complex $K$, and written $S R_{Q}(K)$. This ring is a fascinating invariant of $K$, and reflects many of its combinatorial and geometrical properties, as explained in [33]. Its $Q$-dual is a graded incidence coalgebra [20], which we denote by $S R^{Q}(K)$.

We define a $\operatorname{CAT}(K)^{o p}$-diagram $D_{K}$ in TOP + as follows. The value of $D_{K}$ on each face $\sigma$ is the discrete space $\sigma_{+}$, obtained by adjoining + to the vertices, and the value on $\tau \supseteq \sigma$ is the projection $\tau_{+} \rightarrow \sigma_{+}$, which fixes the vertices of $\sigma$ and maps the vertices of $\tau \backslash \sigma$ to + .

Definition 3.8. Given objects $(X, *)$ of $\mathrm{TOP}_{+}$and $M$ of $\mathrm{TMG}$, the exponential diagrams $X^{K}$ and $M^{K}$ are the cotensor homsets $\operatorname{Hom}_{\mathrm{ID}}\left(D_{K}, X\right)$ and $\operatorname{Hom}_{\mathrm{ID}}\left(D_{K}, M\right)$ respectively; they are $\mathrm{CAT}(K)$-diagrams in $\mathrm{TOP}_{+}$and TMG. Alternatively, they are the respective compositions of the exponentiation functors $X^{()}: \mathrm{TOP}_{+}^{o p} \rightarrow \mathrm{TOP}_{+}$and $M^{()}$: $\mathrm{TOP}_{+}^{o p} \rightarrow \mathrm{TMG}$ with $D_{K}^{o p}$.

So the value of $X^{K}$ on each face $\sigma$ is the product space $X^{\sigma}$, whose elements are functions $f: \sigma \rightarrow X$, and the value of $X^{K}$ on $\sigma \subseteq \tau$ is the inclusion $X^{\sigma} \subseteq X^{\tau}$ obtained by extending $f$ over $\tau$ by the constant map $*$. The space $X^{\varnothing}$ consists only of $*$. In the case of $M^{K}$, each $M^{\sigma}$ is invested with pointwise multiplication, so $H^{K}$ takes values in GRP for a discrete group $H$.

In $\mathrm{G}_{Q} \mathrm{CALG}$, we define a $\operatorname{CAT}(K)^{o p}$-diagram $Q[K]$ by analogy. Its value on $\sigma$ is the graded polynomial algebra $Q[\sigma]$, and on $\tau \supseteq \sigma$ is the projection $Q[\tau] \rightarrow Q[\sigma]$. We denote the dual $\operatorname{CAT}(K)$-diagram $\operatorname{Hom}_{\mathrm{ID}}(Q[K], Q)$ by $Q\langle K\rangle$, and note that it lies in $\mathrm{G}_{Q}$ COCOA. Its value on $\sigma$ is the free $Q$-module $Q\langle S(\sigma)\rangle$ generated by simplices $z$ in $S(\sigma)$, and on $\sigma \subseteq \tau$ is the corresponding inclusion of coalgebras. The coproduct is given by $\delta(z)=\sum z_{1} \otimes z_{2}$, where the sum ranges over all partitions of $z$ into subsimplices $z_{1}$ and $z_{2}$.

When $Q=\mathbb{Z} / 2$ we let the vertices have dimension 1 . Every monomial $v_{U}$ therefore has dimension $|U|$ in the graded algebra $\mathbb{Z} / 2[\sigma]$, and every $j$-simplex in $S(\sigma)$ has dimension $j+1$ in $\mathbb{Z} / 2\langle S(\sigma)\rangle$. We refer to this as the real case. When $Q=\mathbb{Z}$ we consider two possibilities. First is the complex case, in which the vertices have dimension 2 , so that the additive generators of $\mathbb{Z}[\sigma]$ and $\mathbb{Z}\langle S(\sigma)\rangle$ have twice the dimension of their real counterparts. Second is the exterior case, in which the dimension of the vertices reverts to 1 . Every squarefree monomial $v_{U}$ then has dimension $|U|$ in $\mathbb{Z}[\sigma]$, and anticommutativity ensures that every monomial containing a square is zero; every $j$-face of $\sigma$ has dimension $j+1$ in $\mathbb{Z}\langle S(\sigma)\rangle$, and every degenerate $j$-simplex $z$ represents zero. To distinguish between the complex and exterior cases, we write $Q$ as $\mathbb{Z}$ and $\wedge$ respectively.

In the real and complex cases, Davis and Januszkiewicz [12] introduce homotopy types $D J_{\mathbb{R}}(K)$ and $D J_{\mathbb{C}}(K)$. The cohomology rings $H^{*}\left(D J_{\mathbb{R}}(K) ; \mathbb{Z} / 2\right)$ and $H^{*}\left(D J_{\mathbb{C}}(K) ; \mathbb{Z}\right)$ are isomorphic to the graded Stanley-Reisner algebras $S R_{\mathbb{Z} / 2}(K)$ and $S R_{\mathbb{Z}}(K)$ respectively. We shall deal with the exterior case below, and discuss alternative constructions for all three cases. We write $D J(K)$ as a generic symbol for Davis and Januszkiewicz's homotopy types, and refer to them as Davis-Januszkiewicz spaces for $K$. They are represented by objects in TOP. 


\section{Colimits}

In this section we introduce the colimits which form our main topic of discussion, appealing to the completeness and cocompleteness of T and TMG as described in Section 2. We consider colimits of the diagrams $X^{K}, M^{K}, G^{K}$, and $Q\langle K\rangle$ in the appropriate categories, and label them $\operatorname{colim}^{+} X^{K}$, colim ${ }^{\mathrm{TMG}} M^{K}$, $\operatorname{colim}^{\mathrm{TMG}} G^{K}$, and colim $Q\langle K\rangle$ respectively. Similarly, we write the limit of $Q[K]$ as $\lim Q[K]$. As we shall see, these limits and colimits coincide with familiar constructions in several special cases.

As an exercise in acclimatisation, we begin with the diagrams associated to (3.3). Exponentiating with respect to $(X, *)$ and taking colimits provides the chain of subspaces

$$
\bigvee_{j=1}^{m} X_{j} \longrightarrow \operatorname{colim}^{+} X^{K} \longrightarrow X^{m},
$$

thereby sandwiching colim ${ }^{+} X^{K}$ between the axes and the cartesian power. On the other hand, using an object $M$ of TMG yields the chain of epimorphisms

$$
\underset{j=1}{\stackrel{m}{*}} M_{j} \longrightarrow \operatorname{colim}^{\mathrm{TMG}} M^{K} \longrightarrow M^{m}
$$

giving a presentation of $\operatorname{colim}^{\mathrm{TMG}} M^{K}$ which lies between the $m$-fold free product of $M$ and the cartesian power.

The following example emphasises the influence of the underlying category on the formation of colimits, and is important later.

Example 4.3. If $K$ is the non-flag complex $\partial(m)$ of Example 3.2 (where $m>2$ ), then colim $^{+} X^{K}$ is the fat wedge subspace $\left\{\left(x_{1}, \ldots, x_{m}\right): x_{j}=*\right.$ for some $\left.1 \leq j \leq m\right\}$; on the other hand, colim ${ }^{\mathrm{TMG}} M^{K}$ is isomorphic to $M^{m}$ itself.

By construction, colim ${ }^{\mathrm{TMG}} C_{2}^{K}$ in GRP enjoys the presentation

$$
\left\langle a_{1}, \ldots, a_{m}: a_{j}^{2}=1,\left(a_{i} a_{j}\right)^{2}=1 \text { for all }\left\{v_{i}, v_{j}\right\} \text { in } K\right\rangle
$$

and is isomorphic to the right-angled Coxeter group Cox $\left(K^{(1)}\right)$ determined by the 1-skeleton of $K$. Readers should not confuse $K^{(1)}$ with the more familiar Coxeter graph of the group, which is almost its complement!

Similarly, colim ${ }^{\mathrm{TMG}} C^{K}$ has the presentation

$$
\left\langle b_{1}, \ldots, b_{m}:\left[b_{i}, b_{j}\right]=1 \text { for all }\left\{v_{i}, v_{j}\right\} \text { in } K\right\rangle
$$

(where $\left[b_{i}, b_{j}\right]$ denotes the commutator $b_{i} b_{j} b_{i}^{-1} b_{j}^{-1}$ ), and so is isomorphic to the right-angled Artin group $\operatorname{Art}\left(K^{(1)}\right)$. Such groups are sometimes called graph groups, and are special examples of graph products [10]. As explained to us by Dave Benson, neither should be confused with the graphs of groups described in [31].

In the continuous case, we define the circulation group $\operatorname{Cir}\left(K^{(1)}\right)$ as $\operatorname{colim}^{\text {TMG }} T^{K}$ in TMG. Every element of $\operatorname{Cir}\left(K^{(1)}\right)$ may therefore be represented as a word

$$
t_{i_{1}}(1) \cdots t_{i_{k}}(k),
$$

where $t_{i_{j}}(j)$ lies in the $i_{j}$ th factor $T_{i_{j}}$ for each $1 \leq j \leq k$. Two elements $t_{r} \in T_{r}$ and $t_{s} \in T_{s}$ commute whenever $\{r, s\}$ is an edge of $K$.

We shall use $G$ as a generic symbol for any one of the groups $C_{2}, C$, or $T$.

Following (4.2), we abbreviate the generating subgroups $G^{v_{j}}<\operatorname{colim} G^{K}$ to $G_{j}$, where $1 \leq j \leq m$, and call them the vertex groups. Since colim ${ }^{\mathrm{TMG}} G^{K}$ is presented as a quotient of 
the free product $*_{j=1}^{m} G_{j}$, its elements $g$ may be assigned a wordlength $l(g)$. In addition, the arguments of [8] apply to decompose every $g$ from the right as

$$
g=\prod_{j=1}^{n} s_{j}(g)
$$

for some $n \leq l(g)$, where each subword $s_{j}(g)$ contains the maximum possible number of mutually commuting letters, and is unique.

Given any subset $W \subseteq V$ of vertices, we write $K_{W}$ for the complex obtained by restricting $K$ to $W$. The following Lemma is a simple restatement of the basic properties of $\operatorname{colim}^{\mathrm{TMG}} G^{K}$.

Lemma 4.6. We have that

(1) the subgroup colim ${ }^{\mathrm{TMG}} G^{K_{W}} \leq \operatorname{colim}^{\mathrm{TMG}} G^{K}$ is abelian if and only if $K_{W}^{(1)}$ is a complete graph, in which case it is isomorphic to $G^{W}$;

(2) when $K$ is flag, each subword $s_{j}(g)$ of (4.5) lies in a subgroup $G^{\sigma_{j}}$ for some face $\sigma_{j}$ of $K$.

Other algebraic examples of our colimits relate to the Stanley-Reisner algebras and coalgebras of $K$. By construction, there are algebra isomorphisms

$$
\lim \mathbb{Z} / 2[K] \cong S R_{\mathbb{Z} / 2}(K), \quad \lim \mathbb{Z}[K] \cong S R_{\mathbb{Z}}(K), \quad \text { and } \quad \lim \wedge[K] \cong S R_{\wedge}(K),
$$

where the limits are taken in $\mathrm{G}_{\mathbb{Z}} \mathrm{CALG}$. Dually, there are coalgebra isomorphisms

$$
\operatorname{colim} \mathbb{Z} / 2\langle K\rangle \cong S R^{\mathbb{Z} / 2}(K), \quad \operatorname{colim} \mathbb{Z}\langle K\rangle \cong S R^{\mathbb{Z}}(K), \quad \text { and } \quad \operatorname{colim} \wedge\langle K\rangle \cong S R^{\wedge}(K)
$$

in $\mathrm{G}_{\mathbb{Z}} \mathrm{COCOA}$. The analogues of 4.1 display these limits and colimits as

$$
\bigoplus_{j=1}^{m} Q\left[v_{j}\right] \longleftarrow \lim Q[K] \longleftarrow Q[V] \text { and } \bigoplus_{j=1}^{m} D P^{Q}\left(v_{j}\right) \longrightarrow \operatorname{colim} Q\langle K\rangle \longrightarrow D P^{Q}(V)
$$

respectively; here $D P^{Q}(W)$ denotes the divided power $Q$-coalgebra of multisets on $W \subseteq V$, graded by dimension.

If we let $(X, *)$ be one of the pairs $\left(B C_{2}, *\right),(B T, *)$, or $(B C, *)$, then simple arguments with cellular chain complexes show that the cohomology rings $H^{*}\left(\operatorname{colim}^{+}\left(B C_{2}\right)^{K} ; \mathbb{Z} / 2\right)$, $H^{*}\left(\operatorname{colim}^{+}(B T)^{K} ; \mathbb{Z}\right)$, and $H^{*}\left(\operatorname{colim}^{+}(B C)^{K} ; \mathbb{Z}\right)$ are isomorphic to the limits (4.7) respectively. Similarly, the homology coalgebras are isomorphic to the dual coalgebras (4.8). In cohomology, these observations are due to Buchstaber and Panov [7] in the real and complex cases, and to Kim and Roush [22] in the exterior case (at least when $K$ is 1-dimensional). In homology, they may be made in the context of incidence coalgebras, following [29]. In both cases, the maps of (4.1) induce the homomorphisms (4.9).

Such calculations do not themselves identify colim ${ }^{+}\left(B C_{2}\right)^{K}$ and $\operatorname{colim}^{+}(B T)^{K}$ with Davis and Januszkiewicz's constructions. Nevertheless, Buchstaber and Panov provide homotopy equivalences colim ${ }^{+}\left(B C_{2}\right)^{K} \simeq D J_{\mathbb{R}}(K)$ and $\operatorname{colim}^{+}(B T)^{K} \simeq D J_{\mathbb{C}}(K)$, which also follow from Corollary 5.3 below; the Lemma yields a corresponding equivalence in the exterior case. Of course, $\operatorname{colim}^{+}(B C)^{K}$ is a subcomplex of the $m$-dimensional torus $\left(S^{1}\right)^{m}$, and is therefore finite.

In due course, we shall use these remarks to interpret the following proposition in terms of Davis-Januszkiewicz spaces. The proof for $G=C_{2}$ is implicit in [12], and for $G=C$ is due to Kim and Roush [22]. 
Proposition 4.10. When $G=C_{2}$ or $C$, there is a homotopy equivalence

$$
\operatorname{colim}^{+}(B G)^{K} \simeq B \operatorname{colim}^{\mathrm{TMG}} G^{K}
$$

for any flag complex $K$.

Since both cases are discrete, $B$ colim $^{\text {TMG }} G^{K}$ is, of course, an Eilenberg-Mac Lane space; Charney and Davis [9] have since identified good models for $B A$, given any Artin group $A$. Proposition 4.10 fails for arbitrary complexes $K$, as our next examples show.

Examples 4.11. Proposition 4.10 applies when $K=V$, because the discrete complex is flag; then colim $^{\mathrm{TMG}} G^{K}$ is isomorphic to the free product of $m$ copies of $G$, whose classifying space is the $m$-fold wedge $\bigvee_{j=1}^{m} B G_{j}$ (by [6], for example). On the other hand, when $K$ is the non-flag complex $\partial(m)$, Example 4.3 confirms that $B$ colim $^{\mathrm{TMG}} G^{K}$ is $B G^{m}$, whereas $\operatorname{colim}^{+}(B G)^{K}$ is the fat wedge subspace.

These examples apply unchanged to the case $G=T$, and serve to motivate our extension of Proposition 4.10 to the complex case in Proposition 6.1 below. So far as $C_{2}$ and $C$ are concerned, the Proposition asserts that certain homotopy homomorphisms

$$
h_{K}: \Omega \operatorname{colim}^{+}(B G)^{K} \longrightarrow \operatorname{colim}^{\mathrm{TMG}} G^{K}
$$

are homotopy equivalences when $K$ is flag. We therefore view the $h_{K}$ as modelling the loop spaces; in the complex case, they express $\Omega \operatorname{colim}^{+}(B T)^{K}$ in terms of the circulation groups colim $^{\text {TMG }} T^{K}$. In Section 7 we will use homotopy colimits to describe analogues of $h_{K}$ for all complexes $K$.

Our interest in the loop spaces $\Omega \operatorname{colim}^{+}(B G)^{K}$ has been stimulated by several ongoing programmes in combinatorial algebra. For example, Herzog, Reiner, and Welker [17] discuss combinatorial issues associated with calculating the $k$-vector spaces $\operatorname{Tor}^{S R_{k}(K)}(k, k)$ over an arbitrary ground field $k$, and refer to [16] for historical background. Such calculations have applications to diagonal subspace arrangements, as explained by Peeva, Reiner and Welker [28]. Since these Tor spaces also represent the $E_{2}$-term of the Eilenberg-Moore spectral sequence for $H^{*}(\Omega D J(K) ; k)$, it seems well worth pursuing geometrical connections. We consider the algebraic implications elsewhere [27].

\section{Fibrations and homotopy Colimits}

In this section we apply the theory of homotopy colimits to study various relevant fibrations and their geometrical interpretations. Some of the results appear in [7], but we believe that our approach offers an attractive and efficient alternative, and eases generalisation. We refer to [18] and [36] for the notation and fundamental properties of homotopy colimits. Several of the results we use are also summarised in [37], together with additional information on combinatorial applications.

We begin with a general construction, based on a well-pointed topological group $\Gamma$ and a diagram $H: \mathrm{A} \rightarrow$ TMG of closed subgroups and their inclusions. We assume that the maps of the classifying diagram $B H: \mathrm{A} \rightarrow \mathrm{TOP}_{+}$are cofibrations, and that the Projection Lemma [37] applies to the natural projection hocolim ${ }^{+} B H \rightarrow \operatorname{colim}^{+} B H$, which is therefore a homotopy equivalence. The cofibrations $B H(a) \rightarrow B \Gamma$ correspond to the canonical map $f_{H}: \operatorname{colim}^{+} B H \rightarrow B \Gamma$ under the homeomorphism (2.9).

By Examples 2.1 the coset spaces $\Gamma / H(a)$ define an $\mathrm{A} \times \mathrm{C}(\Gamma)$ diagram $\Gamma / H$ in Top, and by Examples 2.20 the cofibration $B H(a) \rightarrow B \Gamma$ is equivalent to the fibration

$$
B\left(*, \mathrm{C}(\Gamma), C(\Gamma) \times_{\mathrm{C}(\Gamma)} \Gamma / H(a)\right) \longrightarrow B \mathrm{C}(\Gamma)
$$


for each object $a$ of $\mathrm{A}$. So $f_{H}$ is equivalent to

$$
\text { hocolim }^{+} B\left(*, \mathrm{C}(\Gamma), C(\Gamma) \times_{\mathrm{C}(\Gamma)} \Gamma / H\right) \longrightarrow B \Gamma
$$

in the homotopy category of spaces over $B \Gamma$, where the homotopy colimit is taken over A.

Proposition 5.1. The homotopy fibre of $f_{H}$ is the homotopy colimit hocolim ${ }^{+} \Gamma / H$.

Proof. We wish to identify the homotopy fibre of the projection

$$
B\left(*, \mathrm{~A}, B\left(*, \mathrm{C}(\Gamma), C(\Gamma) \times_{\mathrm{C}(\Gamma)} \Gamma / H\right)\right) \longrightarrow B \Gamma .
$$

But we may rewrite the total space as $B(*, \mathrm{~A}, \Gamma / H) \times_{\mathrm{C}(\Gamma)^{o p}} B(*, \mathrm{C}(\Gamma), C(\Gamma))$, and therefore as $B(*, \mathrm{C}(\Gamma), C(\Gamma)) \times_{\mathrm{C}(\Gamma)} B(*, \mathrm{~A}, \Gamma / H)$, using (2.19) and Examples 2.20. So the homotopy fibre is $B(*, \mathrm{~A}, \Gamma / H)$, as required.

Given a pair of simplicial complexes $(L, K)$ on vertices $V$, we let $\mathrm{A}=\mathrm{CAT}(K)$, and choose $\Gamma=\operatorname{colim}^{\mathrm{TMG}} G^{L}$ and $H=G^{K}$; we also abbreviate the diagram $\Gamma / H$ to $L / K$. Then $f_{H}$ is the induced map

$$
f_{K, L}: \operatorname{colim}^{+}(B G)^{K} \longrightarrow B \operatorname{colim}^{\mathrm{TMG}} G^{L},
$$

and the Projection Lemma applies to $(B G)^{K}$ because the maps colim ${ }^{+}(B G)^{K \Downarrow \sigma} \rightarrow B G^{\sigma}$ are closed cofibrations for each face $\sigma$. So we have the following corollary to Proposition 5.1.

Corollary 5.3. The homotopy fibre of $f_{K, L}$ is the homotopy colimit hocolim $L / K$, and is homeomorphic to the identification space

$$
\left(B \mathrm{CAT}(K) \times \operatorname{colim}^{\mathrm{TMG}} G^{L}\right) / \sim,
$$

where $(p, g h) \sim(p, g)$ whenever $h \in G^{\sigma}$ and $p$ lies in the face $B(\sigma \downarrow \operatorname{CAT}(K))$.

Proof. By (3.5), the homotopy colimit $B(*, \mathrm{CAT}(K), L / K)$ may be expressed as

$$
B(\downarrow \operatorname{CAT}(K)) \times_{\mathrm{CAT}(K)} L / K,
$$

and the inclusions $B(\sigma \downarrow \operatorname{CAT}(K)) \subseteq B \mathrm{CAT}(K)$ induce a homeomorphism with (5.4).

For future use, we write $\mu$ for the canonical action of $\operatorname{colim}^{\mathrm{TMG}} G^{L}$ on $B(*, \operatorname{CAT}(K), L / K)$.

We note that $f_{K, L}$ coincides with the right-hand map of (4.1) when $L=2^{V}$ and $X=B G$; the cases in which $K=L$ (abbreviated to $f_{K}$ ) and $L=F l(K)$ also feature below. The space hocolim $2^{V} / K$ plays a significant rôle in [12], where it is described as the identification space of Corollary 5.3 and denoted by $\mathcal{Z}_{P}$ (with $P$ the dual of $K$, in the sense of Example 3.6). To emphasise this connection, we write hocolim $L / K$ as $\mathcal{Z}_{G}(K, L)$, which we abbreviate to $\mathcal{Z}_{G}(K)$ when $K=L$. It appears repeatedly below, by virtue of Proposition 5.1. Our examples assume that $L=2^{V}$, and continue the theme of Examples 4.11.

Examples 5.5. If $K=V$ then $\mathcal{Z}_{G}\left(K, 2^{V}\right)$ is the homotopy fibre of $\bigvee_{j=1}^{m} B G_{j} \rightarrow B G^{m}$, the inclusion of the axes; it has been of interest to homotopy theorists for many years. If $K$ is the non-flag complex $\partial(m)$, then $\mathcal{Z}_{G}\left(K, 2^{V}\right)$ is homotopy equivalent to $S^{m-1}$ for $G=\mathbb{Z} / 2$, and $S^{2 m-1}$ for $G=T$.

The second of these examples may be understood by noting that the inclusion of the fat wedge in $B G^{m}$ has the Thom complex of the external product $\zeta^{m}$ of Hopf bundles as its cofibre.

Davis and Januszkiewicz [12] prove that the mod 2 cohomology ring of $E C_{2}^{m} \times{ }_{C_{2}^{m}} \mathcal{Z}_{C_{2}}\left(K, 2^{V}\right)$ and the integral cohomology ring of $E T^{m} \times_{T^{m}} \mathcal{Z}_{T}\left(K, 2^{V}\right)$ are isomorphic to the Stanley Reisner algebras $S R_{\mathbb{Z} / 2}^{*}(K)$ and $S R_{\mathbb{Z}}^{*}(K)$ respectively. In view of Corollary 5.3 (in the case $L=2^{V}$ ), 
we regard the spaces colim ${ }^{+}(B G)^{K}$ and the Davis-Januszkiewicz homotopy types as interchangeable from this point on.

The canonical projection $\mathcal{Z}_{G}(K, L) \rightarrow B \mathrm{CAT}(K)$ is obtained by factoring out the action $\mu$ of $\operatorname{colim}^{\mathrm{TMG}} G^{L}$ on hocolim $L / K$. The cubical structure (3.7) of the quotient lifts to an associated decomposition of $\mathcal{Z}_{G}(K, L)$; when $G=T$ and $L=2^{V}$, for example, we recover the description of [7] and [12] in terms of polydiscs and tori.

The action $\mu$ has other important properties.

Proposition 5.6. The isotropy subgroups of $\mu$ are the conjugates $w G^{\sigma} w^{-1}<\operatorname{colim}^{\mathrm{TMG}} G^{L}$, where $\sigma$ ranges over the faces of $K$.

Proof. It suffices to note from Corollary 5.3 that each point $\left[x, w G^{\sigma}\right]$ is fixed by $w G^{\sigma} w^{-1}<$ $\operatorname{colim}^{\mathrm{TMG}} G^{L}$, for any $x \in B(\sigma \downarrow \mathrm{CAT}(K))$.

Corollary 5.7. The commutator subgroup of $\operatorname{colim}^{\mathrm{TMG}} G^{L}$ acts freely on $\mathcal{Z}_{G}(K, L)$ under $\mu$.

Proof. The isotropy subgroups are abelian, and so have trivial intersection with the commutator subgroup.

When $K=L$ and $G=C_{2}$, Proposition 5.6 strikes a familiar chord. The parabolic subgroups of a Coxeter group $H$ are the conjugates $w \Gamma w^{-1}$ of certain subgroups $\Gamma$, generated by subsets of the defining Coxeter system; when $H$ is right-angled, and therefore takes the form $\operatorname{Cox}\left(K^{(1)}\right)$, such subgroups are abelian. When $L=2^{V}$, each subgroup $w G^{\sigma} w^{-1}$ reduces to $G^{\sigma}$. In this case, Proposition 5.6 implies that the isotropy subgroups form an exponential $\mathrm{CAT}^{o p}(K)$-diagram in TGRP, which assigns $G^{\sigma}$ to the face $\sigma$ and the quotient homomorphism $G^{\tau} \rightarrow G^{\sigma}$ to the reverse inclusion $\tau \supseteq \sigma$.

As detailed in [7], the homotopy fibre $\mathcal{Z}_{G}\left(K, 2^{V}\right)$ is closely related to the theory of subspace arrangements and their auxiliary spaces. These spaces are defined in each of the real, complex, and exterior cases, and will feature below; we introduce them here as homotopy colimits.

Given a pointed space $(Y, 0)$, we let $Y_{\times}$denote $Y \backslash 0$. For any subset $W \subseteq V$, we write $Y_{W} \subseteq Y^{V}$ for the coordinate subspace of functions $f: V \rightarrow Y$ for which $f(W)=0$. The set of subspaces

$$
\mathcal{A}_{Y}(K)=\left\{Y_{W}: W \notin K\right\}
$$

is the associated arrangement of $K$, whose complement $U_{Y}(K)$ is given by the equivalent formulae

$$
Y^{V} \backslash \bigcup_{W \notin K} Y_{W}=\left\{f: f^{-1}(0) \in K\right\} .
$$

The CAT $(K)$-diagram $Y(K)$ associates the function space $Y(\sigma)=\left\{f: f^{-1}(0) \subseteq \sigma\right\}$ to each face $\sigma$, and the inclusion $Y(\sigma) \subseteq Y(\tau)$ to each morphism $\sigma \subseteq \tau$. It follows that $Y(\sigma)$ is homeomorphic to $Y^{\sigma} \times\left(Y_{\times}^{V \backslash \sigma}\right)$, and that $U_{Y}(K)$ is colim $Y(K)$.

The exponential CAT $(K)$-diagram $Y_{\times}^{V \backslash K}$ associates $Y_{\times}^{V \backslash \sigma}$ to $\sigma$; when $Y$ is contractible, we may therefore follow Proposition 5.1 by combining the Projection Lemma and Homotopy Lemma of [37] to obtain a homotopy equivalence

$$
\operatorname{hocolim} Y_{\times}^{V \backslash K} \simeq U_{Y}(K) \text {. }
$$

Now let us write $\mathbb{F}$ for one of the fields $\mathbb{R}$ or $\mathbb{C}$. The study of the coordinate subspace arrangements $\mathcal{A}_{\mathbb{F}}(K)$, together with their complements, is a special case of a well-developed theory whose history is rich and colourful (see [2], for example). In the exterior case, we replace $\mathbb{F}$ by the union of a countably infinite collection of 1 -dimensional cones in $\mathbb{R}^{2}$, which 
we call a 1-star and write as $\mathbb{E}$. So $\mathbb{E}^{V}$ is an $m$-star; it is homeomorphic to the union of countably many $m$-dimensional cones in $\left(\mathbb{R}^{2}\right)^{V}$, obtained by taking products.

As $G$ ranges over $C_{2}, T$ and $C$, we let $\mathbb{F}$ denote $\mathbb{R}, \mathbb{C}$ and $\mathbb{E}$ respectively. In all three cases, the natural inclusion of $G$ into $\mathbb{F}_{\times}$is a cofibration, and $\mathbb{F}_{\times}$retracts onto its image. So (5.9) applies, and may be replaced by the corresponding equivalence

$$
\operatorname{hocolim} G^{V \backslash K} \simeq U_{\mathbb{F}}(K) .
$$

Proposition 5.11. The space $\mathcal{Z}_{G}\left(K, 2^{V}\right)$ is homotopy equivalent to $U_{\mathbb{F}}(K)$, for any complex $K$.

Proof. Substitute $L=2^{V}$ in Corollary 5.3 and apply (5.10).

By specialising certain results of [37] and [38], we may also describe $\left(\bigcup_{W \notin K} \mathbb{F}_{W}\right) \backslash 0$ as a homotopy colimit. This space is dual to $U_{\mathbb{F}}(K)$, and appears to have a more manageable homotopy type in many relevant cases. For $G=C_{2}$ and $T$, a version of Proposition 5.11 features prominently in [7].

The following examples illustrate Proposition 5.11, in the light of Examples 5.5.

Examples 5.12. For $m>2$ and $G=T$, the subspace arrangements of the discrete complex $V$ and the non-flag complex $\partial(m)$ are given by

$$
\left\{\left\{z: z_{j}=z_{k}=0\right\}: 1 \leq j<k \leq m\right\} \quad \text { and } \quad\{0\}
$$

respectively; the corresponding complements are

$$
\left\{z: z_{j}=0 \Rightarrow z_{k} \neq 0\right\} \quad \text { and } \quad \mathbb{C}^{m} \backslash 0 .
$$

The former is homotopy equivalent to a wedge of spheres, and the latter to $S^{2 m-1}$.

\section{Flag COMPlexes AND CONNECTIVIty}

In this section, we examine the homotopy fibre $\mathcal{Z}_{G}(K, L)$ more closely. The results form the basis of our model for $\Omega D J(K)$ when $K$ is flag, and enable us to measure the extent of its failure for general $K$.

We consider a flag complex $K$, and substitute $K=L$ into Corollary 5.3 to deduce that $\mathcal{Z}_{G}(K)$ is the homotopy fibre of the cofibration $f_{K}: D J(K) \rightarrow B$ colim $^{\text {TMG }} G^{K}$. It is helpful to abbreviate $B(\sigma \downarrow \mathrm{CAT}(K))$ to $B(\sigma)$ thoughout the following argument.

Proposition 6.1. The cofibration $f_{K}$ is a homotopy equivalence whenever $K$ is flag.

Proof. We prove that $\mathcal{Z}_{G}(K)$ is contractible.

For any face $\sigma \in K$, the space $\left(\operatorname{colim}^{\mathrm{TMG}} G^{K}\right) / G^{\sigma}$ inherits an increasing filtration by subspaces $\left(\operatorname{colim}^{\mathrm{TMG}} G^{K}\right)_{i} / G^{\sigma}$, consisting of those cosets $w G^{\sigma}$ for which a representing element satisfies $l(w) \leq i$. We may therefore define a $\operatorname{CAT}(K)$-diagram $K_{i} / K$, which assigns (colim $\left.{ }^{\mathrm{TMG}} G^{K}\right)_{i} / G^{\sigma}$ to each face $\sigma$ and the corresponding inclusion to each inclusion $\sigma \subseteq \tau$. By construction, $\mathcal{Z}_{G}(K)$ is filtered by the subspaces hocolim $K_{i} / K$ and each inclusion hocolim $K_{i-1} / K \subset$ hocolim $K_{i} / K$ is a cofibration. We proceed by induction on $i$.

For the base case $i=0$, we observe that $\left(\operatorname{colim}^{\mathrm{TMG}} G^{K}\right)_{0} / G^{\sigma}$ is the single point $e G^{\sigma}$ for all values of $\sigma$. Thus hocolim $K_{0} / K$ is homeomorphic to $B(\varnothing)$, and is indeed contractible. To make the inductive step, we assume that hocolim $K_{i} / K$ is contractible for all $i<n$, and write $Q_{n}$ for the quotient space (hocolim $\left.K_{n} / K\right) /\left(\right.$ hocolim $\left.K_{n-1} / K\right)$. It then suffices to prove that $Q_{n}$ is contractible. 
Every point of $Q_{n}$ has the form $\left(x, w G^{\sigma}\right)$, for some $x \in B(\sigma)$ and some $w$ of length $n$. If the final letter of $w$ lies in $G^{\sigma}$, then $\left(x, w G^{\sigma}\right)$ is the basepoint of $Q_{n}$. Otherwise, we rewrite $w$ as $w^{\prime} s$ by (4.5), where $s$ contains the maximum possible number of mutually commuting letters. These determine a subset $\chi \subseteq V$, and Lemma 4.6 confirms that $K^{(1)}$ contains the complete graph on vertices $\chi$. Since $K$ is flag, we deduce that $2^{\chi} \in K$, and therefore that $\left(x, w^{\prime} G^{\chi}\right)$ is the basepoint of $Q_{n}$. To describe a contraction of $Q_{n}$, we may find a canonical path $p$ in $\operatorname{CAT}_{\varnothing}(K)$, starting at $x$ and finishing at some $x^{\prime}$ in $B(\chi)$; of course $p$ must vary continuously with $\left(x, w G^{\sigma}\right)$, and lift to a corresponding path in $Q_{n}$. If $x$ is a vertex of $B(\sigma)$, we choose $p$ to run at constant speed along the edge from $x$ to the cone point $\varnothing$, and again from $\varnothing$ to the vertex $\chi \in B(\chi)$. If $x$ is an interior point of $B(\sigma)$, we extend the construction by linearity. Then $p$ lifts to the path through $(p(t), w)$ for all $0<t<1$, as required.

Proposition 6.1 leads to the study of $f_{K, L}: D J(K) \rightarrow B$ colim $^{\mathrm{TMG}} G^{L}$ for any subcomplex $K \subseteq L$. We consider the missing faces of $K$ with three or more vertices and write $c(K) \geq 2$ for their minimal dimension. We let $d(K)$ denote $c(K)-1$ when $G=C_{2}$ or $C$, and $2 c(K)$ when $G=T$; thus $K$ is flag if and only if $c(K)$ (and therefore $d(K)$ ) is infinite. Finally, we define

$$
c(K, L)= \begin{cases}c(K) & \text { if } L \subseteq F l(K) \\ 1 & \text { otherwise, }\end{cases}
$$

and let $d(K, L)$ be given by $c(K, L)-1$ or $2 c(K, L)$ as before.

Theorem 6.2. For any subcomplex $K \subseteq L$, the cofibration $f_{K, L}$ is a $d(K, L)$-equivalence.

Proof. We may factorise $f_{K, L}$ as

$$
D J(K) \longrightarrow D J(F l(K)) \longrightarrow D J(F l(L)) \longrightarrow B \operatorname{colim}^{\mathrm{TMG}} G^{F l(L)} .
$$

The first map is induced by flagification, and is a $d(K)$-equivalence by construction. The second is the identity if $L \subseteq F l(K)$; otherwise, it is 0 -connected when $G=C_{2}$ or $C$, and 2connected when $G=T$. The third map is $f_{F l(L)}$, and an equivalence by Proposition 6.1.

Theorem 6.2 suggests our first model for $\Omega D J(K)$.

Proposition 6.3. There is a homotopy homomorphism $h_{K}: \Omega D J(K) \rightarrow \operatorname{colim}^{\mathrm{TMG}} G^{K}$, which is a $(d(K)-1)$-equivalence for any complex $K$; in particular, it is an equivalence if $K$ is flag.

Proof. Applying Theorem 6.2 with $K=L$ implies that $\Omega f_{K}: \Omega D J(K) \rightarrow \Omega B$ colim $^{\text {TMG }} G^{K}$ is a $(d(K)-1)$-equivalence. The result follows by composing with the canonical homotopy homomorphism $\Omega B H \rightarrow H$, which exists for any topological group $H$.

When $L=2^{V}$, the missing faces of $\left(2^{V}, K\right)$ are precisely the non-faces of $K$. In this case only, we write their minimal dimension as $c^{\prime}(K)$.

It is instructive to consider the homotopy commutative diagram

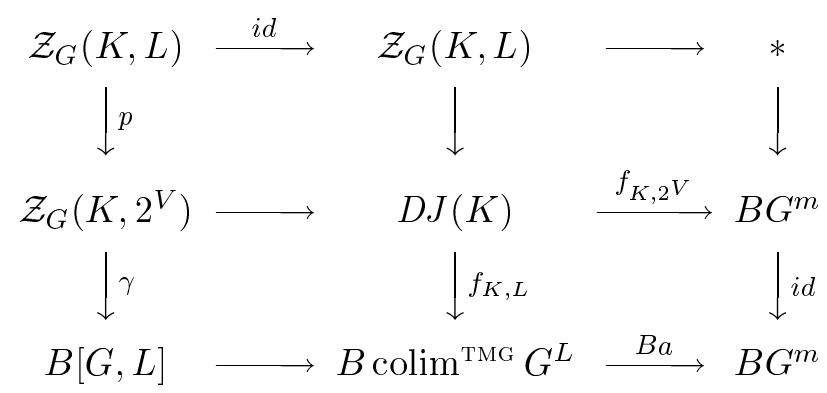


of fibrations, where $a$ is the abelianisation homomorphism and $[G, L]$ denotes the commutator subgroup of $\operatorname{colim}^{\mathrm{TMG}} G^{L}$. By Theorem $6.2, \mathcal{Z}_{G}(K, L)$ and $\mathcal{Z}_{G}\left(K, 2^{V}\right)$ are $(d(K, L)-1)$ - and $\left(d^{\prime}(K)-1\right)$-connected respectively, where $d(K, L) \geq d^{\prime}(K)$ by definition. In fact $\mathcal{Z}_{G}\left(K, 2^{V}\right)$ is $d^{\prime}(K)$-connected, by considering the homotopy exact sequence of $f_{K, 2^{V}}$.

Corollary 5.7 confirms that

$$
[G, L] \longrightarrow \mathcal{Z}_{G}(K, L) \stackrel{p}{\longrightarrow} \mathcal{Z}_{G}\left(K, 2^{V}\right)
$$

is a principal $[G, L]$-bundle, classified by $\gamma$. This bundle encodes a wealth of geometrical information on the pair $(L, K)$. Its total space measures the failure of $f_{K, L}$ to be a homotopy equivalence, and its base space is the complement of the coordinate subspace arrangement $\mathcal{A}_{\mathbb{F}}(K)$ by Corollary 5.11. Moreover, Theorem 6.2 implies that $\gamma$ is also a $d(K, L)$-equivalence, and so sheds some light on the homotopy type of $U_{\mathbb{F}}(K)$.

Looping (6.4) gives a homotopy commutative diagram of fibrations



in TMONH, which offers an alternative perspective on $\Omega D J(K)$.

Lemma 6.7. The loop space $\Omega D J(K)$ splits as $G^{m} \times \Omega U_{\mathbb{F}}(K)$ for any simplicial complex $K$; the splitting is not multiplicative.

Proof. The vertex groups $G_{j}$ embed in $\Omega D J(K)$ via homotopy homomorphisms, whose product $j: G^{m} \rightarrow \Omega D J(K)$ is left inverse to $\Omega f_{K, 2^{V}}$ (but not a homotopy homomorphism). The product of the maps $i$ and $j$ is the required homeomorphism.

The following examples continue the theme of Examples 5.5 and 5.12. They refer to the second horizontal fibration of the diagram (6.6), which is homotopy equivalent to the third whenever $K=L$ is flag, by Proposition 6.1. The second examples also appeal to James's Theorem [19], which identifies the loop space $\Omega S^{n}$ with the free monoid $F^{+}\left(S^{n-1}\right)$ for any $n>1$.

Examples 6.8. If $K$ is the discrete flag complex $V$, then $\Omega U_{\mathbb{F}}(K)$ is homotopy equivalent to the commutator subgroup of the free product $*_{j=1}^{m} G_{j}$. If $K$ is the non-flag complex $\partial(m)$, then $\Omega U_{\mathbb{F}}(K)$ is homotopy equivalent to $F^{+}\left(S^{m-2}\right)$ for $G=\mathbb{Z} / 2$, and $F^{+}\left(S^{2 m-2}\right)$ for $G=T$; the map $i$ identifies the generators of each free monoid with higher Samelson products (of order m) in $\Omega D J(K)$.

Of course, both examples split topologically according to Lemma 6.7. The appearance of higher products in $\Omega D J(\partial(m))$ shows that commutators alone cannot model $\Omega D J(K)$ when $K$ is not flag. More subtle structures are required, based on higher homotopy commutativity; they are related to Samelson and Whitehead products, as we explain elsewhere [27]. 


\section{Hомоторy COLIMits OF TOPOLOGICAL MONOIDS}

We now turn to the loop space $\Omega D J(K)$ for a general simplicial complex $K$, appealing to the theory of homotopy colimits. Although the resulting models are necessarily more complicated, they are homotopy equivalent to colim ${ }^{\mathrm{TMG}} G^{K}$ when $K$ is flag. The constructions depend fundamentally on the categorical ideas of Section 2, and apply to more general spaces than $D J(K)$. We therefore work with an arbitrary diagram $D:$ A $\rightarrow$ TMG for most of the section, and write $B D: \mathrm{A} \rightarrow \mathrm{TOP}+$ for its classifying diagram. Our applications follow by substituting $G^{K}$ for $D$.

We implement proposals of earlier authors (as in [36], for example) by forming the homotopy colimit hocolim ${ }^{\mathrm{TMG}} D$ in $\mathrm{TMG}$, rather than $\mathrm{TOP}_{+}$. This is made possible by the observation of Section 2 that the categories TMG are T-cocomplete, and therefore have sufficient structure for the creation of internal homotopy colimits. We confirm that hocolim ${ }^{\mathrm{TMG}} D$ is a model for the loop space $\Omega$ hocolim ${ }^{+} B D$ by proving that $B$ commutes with homotopy colimits in the relevant sense. As usual, we work in TMG, but find it convenient to describe certain details in terms of topological monoids; whenever these monoids are topological groups, so is the output.

We recall the standard extension of the 2 -sided bar construction to the based setting, with reference to (2.18). We write $B_{\bullet}^{+}(*, \mathrm{~A}, D)$ for the diagram $\mathrm{B}^{o p} \times \Delta^{o p} \rightarrow \mathrm{TOP}_{+}$given by

$$
(b,(n)) \longmapsto \bigvee_{a_{0}, a_{n}} D\left(b, a_{0}\right) \wedge \mathrm{A}_{n}\left(a_{0}, a_{n}\right)_{+},
$$

where $D$ is a diagram $\mathrm{A} \times \mathrm{B}^{o p} \rightarrow \mathrm{TOP}_{+}$. Following Examples 2.20, we define the homotopy $\mathrm{TOP}_{+}$-colimit as

$$
\operatorname{hocolim}^{+} D=B^{+}(*, \mathrm{~A}, D),
$$

and note the equivalent expressions $B^{+}\left(*, \mathrm{~A}, A_{+}\right) \wedge_{\mathrm{A}} D \cong D \wedge_{\mathrm{A}^{o p}} B^{+}\left(*, \mathrm{~A}, A_{+}\right)$.

For TMG, we proceed by categorical analogy. We replace the TOP-coproduct in (2.18) by its counterpart in TMG, and the internal cartesian product in TOP by the tensored structure of $\mathrm{TMG}$ over $\mathrm{TOP}_{+}$. For any diagram $D: \mathrm{A} \rightarrow \mathrm{TMG}$, the simplicial topological monoid $B_{\bullet}^{\text {TMG }}(*, \mathrm{~A}, D)$ is therefore given by

$$
(n) \longmapsto \underset{a_{0}, a_{n}}{*} D\left(a_{0}\right) \circledast \mathrm{A}_{n}\left(a_{0}, a_{n}\right)_{+},
$$

where $*$ denotes the free product of topological monoids. The face and degeneracy operators are defined as before, but are now homomorphisms. When $\mathrm{A}$ is of the form $\operatorname{CAT}(K)$, the $n$-simplices (7.1) may be rewritten as the finite free product

$$
B_{n}^{\mathrm{TMG}}(*, \operatorname{CAT}(K), D)=\underset{\sigma_{n} \supseteq \cdots \supseteq \sigma_{0}}{*} D\left(\sigma_{0}\right),
$$

where there is one factor for each $n$-chain of simplices in $K$.

Definition 7.2. The homotopy TMG-colimit of $D$ is given by

$$
\operatorname{hocolim}^{\mathrm{TMG}} D=\left|B_{\bullet}^{\mathrm{TMG}}(*, \mathrm{~A}, D)\right|_{\mathrm{TMG}}
$$

in TMG, for any diagram $D: \mathrm{A} \rightarrow$ TMG.

So hocolim ${ }^{\mathrm{TMG}} D$ is an object of TMG. Following Construction (2.11), it may be described in terms of generators and relations as a quotient monoid of the form

$$
\left(\underset{n \geq 0}{*}\left(B_{n}(*, \mathrm{~A}, D) \circledast \Delta_{+}^{n}\right)\right) /\left\langle\left(d_{n}^{i}(b), s\right)=\left(b, \delta_{n}^{i}(s)\right),\left(s_{n}^{i}(b), t\right)=\left(b, \sigma_{n}^{i}(t)\right)\right\rangle,
$$


for all $b \in B_{n}(*, \mathrm{~A}, D)$, and all $s \in \Delta(n-1)$ and $t \in \Delta(n+1)$. Here $\delta_{n}^{i}$ and $\sigma_{n}^{i}$ are the standard face and degeneracy maps of geometric simplices.

Example 7.3. Suppose that A is the category $\cdot \rightarrow \cdot$, with a single non-identity. Then an Adiagram is a homomorphism $M \rightarrow N$ in TMG, and hocolim ${ }^{\mathrm{TMG}} D$ is its TMG mapping cylinder. It may be identified with the TMG-pushout of the diagram

$$
M \circledast \Delta(1)_{+} \stackrel{j}{\longleftarrow} M \longrightarrow N,
$$

where $j(m)=(m, 0)$ in $M \circledast \Delta(1)_{+}$for all $m \in M$.

An alternative expression for the simplicial topological monoid $B_{\bullet}^{\mathrm{TMG}}(*, \mathrm{~A}, D)$ arises by analogy with the equivalences (2.19).

Proposition 7.4. There is an isomorphism $D \circledast_{\mathrm{A}^{o p}} B_{\bullet}^{+}\left(*, \mathrm{~A}, A_{+}\right) \cong B_{\bullet}^{\mathrm{TMG}}(*, \mathrm{~A}, D)$ of simplicial topological monoids, for any diagram $D: \mathrm{A} \rightarrow$ TMG.

Proof. By $(2.16)$, the functor $D \circledast_{\mathrm{A}^{o p}}:\left[\mathrm{A}^{o p} \times \Delta^{o p}, \mathrm{TOP}_{+}\right] \rightarrow\left[\Delta^{o p}, \mathrm{TMG}\right]$ is left $\mathrm{TOP}_{+}$-adjoint to $\operatorname{TMG}(D$,$) , and therefore preserves coproducts. So we may write$

$$
\begin{aligned}
D \circledast_{\mathrm{A}^{o p}} B_{\bullet}^{+}\left(*, \mathrm{~A}, A_{+}\right) & \cong \underset{a, b}{*} D \circledast_{\mathrm{A}^{o p}}\left(\mathrm{~A}(, a)_{+} \wedge \mathrm{A}_{\bullet}(a, b)_{+}\right) \\
& \cong \underset{a, b}{*} D(a) \circledast \mathrm{A}_{\bullet}(a, b)_{+}
\end{aligned}
$$

as required, using the isomorphism $D \circledast \mathrm{A}^{o p} \mathrm{~A}(, a) \cong D(a)$ of $(2.15)$.

It is important to establish when the simplicial topological monoids $B_{\bullet}^{\mathrm{TMG}}(*, \mathrm{~A}, D)$ are proper simplicial spaces, in the sense of [25], because we are interested in the homotopy type of their realisations. This is achieved in Proposition 7.8, and leads on to the analogue of the Homotopy Lemma for TMG. These are two of the more memorable of the following sequence of six preliminaries, which precede the proof of our main result. On several occasions we insist that objects of TMG are well-pointed, and even that they have the homotopy type of a $\mathrm{CW}$-complex. Such conditions certainly hold for our exponential diagrams, and do not affect the applications.

We consider families of monoids indexed by the elements $s$ of an arbitrary set $S$.

Lemma 7.5. Let $f_{s}: M_{s} \rightarrow N_{s}$ be a family of homomorphisms of well-pointed monoids, which are homotopy equivalences; then the coproduct homomorphism

$$
\underset{s}{*} f_{s}: \underset{s}{*} M_{s} \longrightarrow \underset{s}{*} N_{s}
$$

is also a homotopy equivalence.

Proof. Let $f: M \rightarrow N$ denote the homomorphism in question, and write $F_{k} M$ for the subspace of $M$ of elements representable by words of length $\leq k$. Hence $F_{0}=\{e\}$, and $F_{k+1} M$ is the pushout

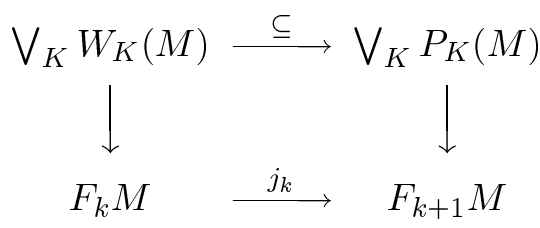

in $\mathrm{TOP}_{+}$, where $K$ runs through all $(k+1)$-tuples $\left(s_{1}, \ldots, s_{k+1}\right) \in S^{k+1}$ such that $s_{i+1} \neq s_{i}$, and $W_{K}(M) \subset P_{K}(M)$ is the fat wedge subspace of $M_{s_{1}} \times \ldots \times M_{s_{k+1}}$. Each $M_{s}$ is well-pointed, so $W_{K}(M) \subset P_{K}(M)$ is a closed cofibration, and therefore so is $j_{k}$. Since $M=\operatorname{colim}_{k} F_{k} M$ in 
$\mathrm{TOP}_{+}$, it remains to confirm that the restriction $f_{k}: F_{k} M \rightarrow F_{k} N$ is a homotopy equivalence for all $k$. We proceed by induction, based on the trivial case $k=0$.

The map $f$ induces a homotopy equivalence $W_{K}(M) \rightarrow W_{K}(N)$ because $M_{s}$ and $N_{s}$ are well-pointed, and a further homotopy equivalence $P_{K}(M) \rightarrow P_{K}(N)$ by construction. So the inductive hypothesis combines with Brown's Gluing Lemma [37, 2.4] to complete the proof.

Lemma 7.7. For any subset $R \subset S$, the inclusion $*_{r} M_{r} \rightarrow{ }_{s} M_{s}$ is a closed cofibration; in particular, $*_{s} M_{s}$ is well-pointed.

Proof. Let $B \rightarrow M$ be the inclusion in question, with $F_{k} M$ as in the proof of Lemma 7.5 and $F_{k}^{\prime} M=B \cup F_{k} M$. Then $F_{k+1}^{\prime} M$ is obtained from $F_{k}^{\prime} M$ by attaching spaces $P_{K}(M)$, where $K$ runs through all $\left(s_{1}, \ldots, s_{k+1}\right)$ in $S^{k+1} \backslash R^{k+1}$ such that $s_{i+1} \neq s_{i}$. Thus $B \rightarrow F_{k}^{\prime} M$ is a cofibration for all $k$, implying the result.

Proposition 7.8. Given any small category $\mathrm{A}$, and any diagram $D: \mathrm{A} \rightarrow \mathrm{TMG}$ of well-pointed monoids, the simplicial space $B_{\bullet}^{\mathrm{TMG}}(*, \mathrm{~A}, D)$ is proper, and its realisation $B^{\mathrm{TMG}}(*, \mathrm{~A}, D)$ is wellpointed.

Proof. By Lemma 7.7, each degeneracy map $B_{n}^{\mathrm{TMG}}(*, \mathrm{~A}, D) \rightarrow B_{n+1}^{\mathrm{TMG}}(*, \mathrm{~A}, D)$ is a closed cofibration. The first result then follows from Lillig's Union Theorem [23] for cofibrations. So $B_{0}^{\mathrm{TMG}}(*, \mathrm{~A}, D) \subset B^{\mathrm{TMG}}(*, \mathrm{~A}, D)$ is a closed cofibration and $B_{0}^{\mathrm{TMG}}(*, \mathrm{~A}, D)$ is well-pointed, yielding the second result.

As described in Examples 2.13, every simplicial object $M_{\bullet}$ in TMG has two possible realisations. We now confirm that they agree, and identify their classifying space.

Lemma 7.9. The realisations $\left|M_{\bullet}\right|_{\mathrm{TMG}}$ and $\left|M_{\bullet}\right|$ are naturally isomorphic objects of $\mathrm{TMG}$, whose classifying space is naturally homeomorphic to $\left|B\left(M_{\bullet}\right)\right|$.

Proof. We apply the techniques of [14, VII §3] and [26, §4] to the functors ||$_{\text {TMG }}$ and the restriction of || to $\left[\Delta^{o p}, \mathrm{TMG}\right]$. Both are left TOP ${ }_{+}$-adjoint to $\operatorname{Sin}: \mathrm{TMG} \rightarrow\left[\Delta^{o p}, \mathrm{TMG}\right]$, and so are naturally equivalent. The homeomorphism $B\left|M_{\bullet}\right| \cong\left|B\left(M_{\bullet}\right)\right|$ arises by considering the bisimplicial object $(k, n) \mapsto\left(M_{n}\right)^{k}$ in TOP + , and forming its realisation in either order.

We may now establish our promised Homotopy Lemma.

Proposition 7.10. Given diagrams $D_{1}, D_{2}: \mathrm{A} \rightarrow \mathrm{TMG}$ of well-pointed topological monoids, and a map $f: D_{1} \rightarrow D_{2}$ such that $f(a): D_{1}(a) \rightarrow D_{2}(a)$ is a homotopy equivalence of underlying spaces for each object a of $\mathrm{A}$, the induced map

$$
\text { hocolim }^{\text {TMG }} D_{1} \longrightarrow \text { hocolim }^{\text {TMG }} D_{2}
$$

is a homotopy equivalence.

Proof. This follows directly from Lemmas 7.5 and 7.9, and Proposition 7.8.

We need one more technical result concerning homotopy limits of simplicial objects. We work with diagrams $X_{\bullet}: \mathrm{A} \times \Delta^{o p} \rightarrow \mathrm{TOP}_{+}$of simplicial spaces, and $D_{\bullet}: \mathrm{A} \times \Delta^{o p} \rightarrow \mathrm{TMG}$ of simplicial topological monoids.

Proposition 7.11. With $X_{\bullet}$ and $D_{\bullet}$ as above, there are natural isomorphisms

$$
\operatorname{hocolim}^{+}\left|X_{\bullet}\right| \cong\left|\operatorname{hocolim}^{+} X_{\bullet}\right| \text { and } \operatorname{hocolim}^{\mathrm{TMG}}\left|D_{\bullet}\right| \cong\left|\operatorname{hocolim}^{\mathrm{TMG}} D_{\bullet}\right|
$$

in $\mathrm{TOP}_{+}$and $\mathrm{TMG}$ respectively. 
Proof. The isomorphisms arise from realising the bisimplicial objects

$$
(k, n) \rightarrow B_{k}^{+}\left(*, \mathrm{~A}, X_{n}\right) \quad \text { and } \quad(k, n) \rightarrow B_{k}^{\mathrm{TMG}}\left(*, \mathrm{~A}, D_{n}\right)
$$

in either order. In the case $D_{\bullet}$, we must also apply the first statement of Lemma 7.9.

Parts of the proofs above may be rephrased using variants of the equivalences (2.15). They lead to our first general result, which states that the formation of classifying spaces commutes with homotopy colimits in an appropriate sense.

Theorem 7.12. For any diagram D: A $\rightarrow$ TMG of well-pointed topological monoids with the homotopy types of $C W$-complexes, the map

$$
g_{D}: \text { hocolim }^{+} B D \longrightarrow B \text { hocolim }^{\text {TMG }} D
$$

is a homotopy equivalence.

Proof. For each object $a$ of A, let $D_{\bullet}(a)$ be the singular simplicial monoid of $D(a)$. The natural map $\left|D_{\bullet}(a)\right| \rightarrow D(a)$ is a homomorphism of well-pointed monoids and a homotopy equivalence, so it passes to a homotopy equivalence $B\left|D_{\bullet}(a)\right| \rightarrow B D(a)$ under the formation of classifying spaces. By Proposition 7.10 and the corresponding Homotopy Lemma for TOP + , it therefore suffices to prove our result for diagrams of realisations of simplicial monoids.

So let $D_{\bullet}: \mathrm{A} \times \Delta^{o p} \rightarrow$ TMG be a diagram of discrete simplicial monoids. By Lemma 7.9 and Proposition 7.11, we must show that the canonical map

$$
\mid \text { hocolim }^{+} B D_{\bullet}|\longrightarrow| B \text { hocolim }^{\text {TMG }} D_{\bullet} \mid
$$

is a homotopy equivalence. Since the simplicial spaces hocolim ${ }^{+} B D \bullet$ and $B$ hocolim ${ }^{\text {TMG }} D_{\bullet}$ are proper, this reduces to proving that

$$
\text { hocolim }^{+} B D_{n} \longrightarrow B \text { hocolim }{ }^{\text {TMG }} D_{n}
$$

is a homotopy equivalence in each dimension. But hocolim ${ }^{+} B D_{n}$ is the realisation of the proper simplicial space $B_{\bullet}^{+}\left(*, \mathrm{~A}, B D_{n}\right)$, and $B$ hocolim $^{\mathrm{TMG}} D_{n}$ is naturally homeomorphic to the proper simplicial space $B\left(B_{\bullet}^{\text {TMG }}\left(*\right.\right.$, A,$\left.\left.D_{n}\right)\right)$ by Lemma 7.9. Moreover, for each $k \geq 0$ the natural map $B_{k}^{+}\left(*, \mathrm{~A}, B D_{n}\right) \rightarrow B\left(B_{k}^{\mathrm{TMG}}\left(*, \mathrm{~A}, D_{n}\right)\right.$ coincides with the map

$$
\bigvee_{a_{0} \rightarrow \cdots \rightarrow a_{k}} B D_{n}\left(a_{0}\right) \longrightarrow B\left(\underset{a_{0} \rightarrow \cdots \rightarrow a_{k}}{*} D_{n}\left(a_{0}\right)\right)
$$

induced by the inclusion of each $D_{n}\left(a_{0}\right)$ into the free product. Since (7.13) is a homotopy equivalence by a theorem of Fiedorowicz $[15,4.1]$, the proof is complete.

Various steps in the proof of Theorem 7.12 may be adapted to verify the following, which answers a natural question about tensored monoids.

Proposition 7.14. For any well-pointed topological monoid $M$ and based space $Y$, the natural map

$$
B M \wedge Y \longrightarrow B(M \otimes Y)
$$

is a homotopy equivalence when $M$ and $Y$ have the homotopy type of $C W$-complexes.

Proof. As in Theorem 7.12, we need only work with the realisations $\left|M_{\bullet}\right|$ and $\left|Y_{\bullet}\right|$ of the total singular complexes. Since $B\left|M_{\bullet}\right| \wedge\left|Y_{\bullet}\right| \rightarrow B\left(\left|M_{\bullet}\right| \otimes\left|Y_{\bullet}\right|\right)$ is the realisation of the natural map $B M_{n} \wedge Y_{n} \rightarrow B\left(M_{n} \otimes Y_{n}\right)$, it suffices to assume that $Y$ is discrete; in this case,

$$
B M \wedge Y \longrightarrow B\left(\underset{y}{*} M_{y}\right)
$$


is a homotopy equivalence by the same result of Fiedorowicz [15].

We apply Theorem 7.12 to construct our general model for $\Omega D J(K)$, but require a commutative diagram to clarify its relationship with the special case $h_{K}$ of Proposition 6.3. We deal with $\mathrm{A}^{o p} \times \Delta^{o p}$-diagrams $X_{\bullet}$ in $\mathrm{TOP}_{+}$, and certain of their morphisms. These include $\theta: X_{\bullet} \rightarrow \mathrm{TOP}_{+}\left(B D, B\left(D \circledast_{\mathrm{A}^{o p}} X_{\bullet}\right)\right)$, defined for any $X_{\bullet}$ by $\theta(x)=B(d \mapsto d \circledast x)$, and the projection $\pi: B_{\bullet}^{+} \rightarrow\left(*_{+}\right) \bullet$, where $B_{\bullet}^{+}$and $\left(*_{+}\right) \bullet$ denote $B_{\bullet}^{+}(*, \mathrm{~A}, A)$ and the trivial diagram respectively. Under the homeomorphism

$$
\left[\Delta^{o p}, \mathrm{TOP}_{+}\right]\left(B D \wedge_{\mathrm{A}^{o p}} X_{\bullet}, B\left(D \circledast_{\mathrm{A}^{o p}} X_{\bullet}\right)\right) \cong\left[\mathrm{A}^{o p} \times \Delta^{o p}, \mathrm{TOP}_{+}\right]\left(X_{\bullet}, \mathrm{TOP}_{+}\left(B D, B\left(D \circledast_{\mathrm{A}^{o p}} X_{\bullet}\right)\right)\right)
$$

of $(2.16), \theta$ corresponds to a map $\phi: B D \wedge_{\mathrm{A}^{o p}} X_{\bullet} \rightarrow B\left(D \circledast_{\mathrm{A}^{o p}} X_{\bullet}\right)$ of simplicial spaces.

Proposition 7.15. For any diagram $D: \mathrm{A} \rightarrow \mathrm{TMG}$, there is a commutative square



where $p^{+}$and $p^{\mathrm{TMG}}$ are the natural projections.

Proof. By construction, the diagram



is commutative in $\left[\mathrm{A}^{o p} \times \Delta^{o p}, \mathrm{TOP}_{+}\right]$, and has adjoint

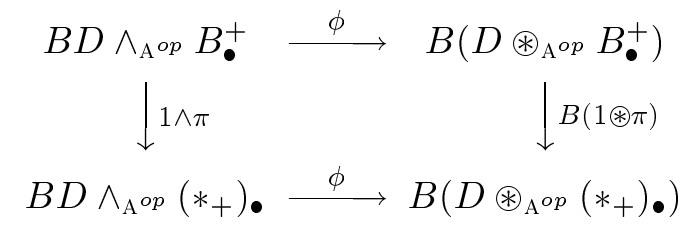

in $\left[\Delta^{o p}, \mathrm{TOP}_{+}\right]$. By Proposition 7.4 , the upper $\phi$ is the map $B_{\bullet}^{+}(*, \mathrm{~A}, B D) \rightarrow B\left(B_{\bullet}^{\mathrm{TMG}}(*, \mathrm{~A}, D)\right)$ obtained by applying the relevant map (7.13) in each dimension. By Examples 2.13, the lower $\phi$ is given by the canonical map $f_{D}: \operatorname{colim}^{+} B D \rightarrow B \operatorname{colim}^{\text {TMG }} D$ in each dimension. Since realisation commutes with $B$, the topological realisation of (7.16) is the diagram we seek; for Lemma 7.9 identifies the upper right-hand space with $B$ hocolim ${ }^{\text {TMG }} D$, and Examples 2.20 confirms that the vertical maps are the natural projections.

Theorem 7.17. There is a homotopy commutative square

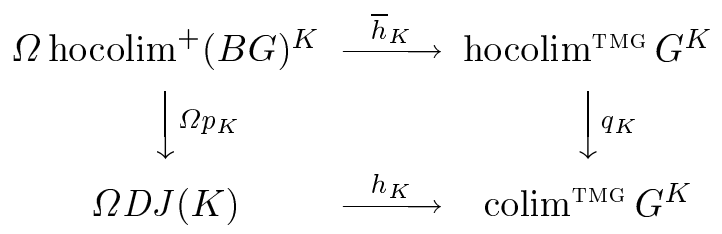

of homotopy homomorphisms, where $p_{K}$ and $\bar{h}_{K}$ are homotopy equivalences for any simplicial complex $K$. 
Proof. We apply Proposition 7.15 with $D=G^{K}$, and loop the corresponding square; the projection $p_{K}$ : hocolim ${ }^{+}(B G)^{K} \rightarrow D J(K)$ is a homotopy equivalence, as explained in Corollary 5.3. The result follows by composing the horizontal maps with the canonical homotopy homomorphism $\Omega B H \rightarrow H$, where $H=\operatorname{hocolim}^{\mathrm{TMG}} G^{K}$ and colim $^{\mathrm{TMG}} G^{K}$ respectively.

It is an interesting challenge to describe good geometrical models for homotopy homomorphisms which are inverse to $h_{K}$ and $\bar{h}_{K}$.

\section{REFERENCES}

[1] Michael Barr and Charles Wells. Toposes, Triples and Theories. Number 278 in Grundlehren der mathematischen Wissenschaften. Springer-Verlag, 1985.

[2] Anders Björner. Subspace arrangements. In A Joseph, F Mignot, F Murat, and B Prum, editors, Proceedings of the First European Congress of Mathematics (Paris 1992), volume 119 of Progress in Mathematics, pages 321-370. Birkhäuser, 1995.

[3] Francis Borceux. Handbook of Categorical Algebra 2, volume 51 of Encyclopedia of Mathematics and its Applications. Cambridge University Press, 1994.

[4] A K Bousfield and Daniel M Kan. Homotopy Limits, Completions and Localizations, volume 304 of Lecture notes in Mathematics. Springer Verlag, 1972.

[5] Michael Brinkmeier. Strongly homotopy-commutative monoids revisited. Documenta Mathematica, 5:613$624,2000$.

[6] Ronald Brown and J P Lewis Hardy. Topological groupoids 1: universal constructions. Mathematische Nachriften, 71:273-286, 1976.

[7] Victor M Buchstaber and Taras E Panov. Torus actions, combinatorial topology, and homological algebra. Russian Mathematical Surveys, 55:825-921, 2000.

[8] Pierre Cartier and Dominique Foata. Problèmes combinatoires de commutation et réarrangements, volume 85 of Lecture Notes in Mathematics. Springer Verlag, 1969.

[9] Ruth Charney and Michael Davis. Finite $K(\pi, 1)$ for Artin groups. In Prospects in Topology (Princeton NJ, 1994), volume 138 of Annals of Mathematics Studies, pages 110-124. Princeton University Press, 1995.

[10] Ian M Chiswell. The Euler characteristic of graph products and of Coxeter groups. In William J Harvey and Colin Maclachlan, editors, Discrete Groups and Geometry (Birmingham, 1991), volume 173 of London Mathematical Society Lecture Note Series, pages 36-46. Cambridge University Press, 1992.

[11] Michael W Davis. Groups generated by reflections and aspherical manifolds not covered by Euclidean space. Annals of Mathematics, 117:293-324, 1983.

[12] Michael W Davis and Tadeusz Januszkiewicz. Convex polytopes, Coxeter orbifolds and torus actions. Duke Mathematical Journal, 62:417-451, 1991.

[13] Albrecht Dold and Richard Lashof. Principal quasifibrations and fibre homotopy equivalence of bundles. Illinois Journal of Mathematics, 3:285-305, 1959.

[14] Anthony D Elmendorf, Igor Kriz, Michael P Mandell, and J Peter May. Rings, Modules, and Algebras in Stable Homotopy Theory, volume 47 of Mathematical Surveys and Monographs. American Mathematical Society, 1997.

[15] Zbigniew Fiedorowicz. Classifying spaces of topological monoids and categories. Journal of the American Mathematical Society, 106:301-350, 1984.

[16] T Gulliksen and G Levin. Homology of local rings, volume 20 of Queen's papers in Pure and applied Mathematics. Queen's University, Kingston, Ontario, 1969.

[17] Jørgen Herzog, Victor Reiner, and Volkmar Welker. Componentwise linear ideals and Golod rings. Michigan Mathematical Journal, 46:211-223, 1999.

[18] Jens Hollender and Rainer M Vogt. Modules of topological spaces, applications to homotopy limits and $E_{\infty}$ structures. Archiv der Mathematik, 59:115-129, 1992.

[19] Ioan M James. Reduced product spaces. Annals of Mathematics, 62:259-280, 1955.

[20] S A Joni and G-C Rota. Coalgebras and bialgebras in combinatorics. Studies in Applied Mathematics, 61:93-139, 1979.

[21] G Maxwell Kelly. Basic Concepts of Enriched Category Theory, volume 64 of London Mathematical Society Lecture Note Series. Cambridge University Press, 1982. 
[22] Ki Hang Kim and Fred W Roush. Homology of certain algebras defined by graphs. Journal of Pure and Applied Algebra, 17:179-186, 1980.

[23] Joachim Lillig. A union theorem for cofibrations. Archiv der Mathematik, 24:410-415, 1973.

[24] J Peter May. Simplicial Objects in Algebraic Topology, volume 11 of Van Nostrand Mathematical Studies. Van Nostrand Reinhold, 1967.

[25] J Peter May. $E_{\infty}$-spaces, group completions and permutative categories. In Graeme Segal, editor, New Developments in Topology, volume 11 of London Mathematical Society Lecture Notes Series, pages 153231. Cambridge University Press, 1974.

[26] James McClure, Roland Schwänzl, and Rainer Vogt. $T H H(R) \cong R \otimes S^{1}$ for $E_{\infty}$ ring spectra. Journal of Pure and Applied Algebra, 121:137-159, 1997.

[27] Taras Panov and Nigel Ray. The homology and homotopy theory of certain loop spaces. In preparation, University of Manchester, 2001.

[28] Irena Peeva, Victor Reiner, and Volkmar Welker. Cohomology of real diagonal subspace arrangments via resolutions. Compositio Mathematica, 117:99-115, 1999.

[29] Nigel Ray and William Schmitt. Combinatorial models for coalgebraic structures. Advances in Mathematics, 138:211-262, 1998.

[30] Roland Schwänzl and Rainer M Vogt. The categories of $A_{\infty}$ and $E_{\infty}$ monoids and ring spectra as closed simplicial and topological model categories. Archiv der Mathematik, 56:405-411, 1991.

[31] Peter Scott and Terry Wall. Topological methods in group theory. In C T C Wall, editor, Homological Group Theory (Proceedings of the Durham Symposium, 1977), volume 36 of London Mathematical Society Lecture Note Series, pages 137-203. Cambridge University Press, 1979.

[32] Graeme Segal. Classifying spaces and spectral sequences. Institut des Hautes Études Scientifiques, Publications Mathematiques, 34:105-112, 1968.

[33] Richard P Stanley. Combinatorics and Commutative Algebra, 2nd edition, volume 41 of Progress in Mathematics. Birkhäuser, Boston, 1996.

[34] Masahiro Sugawara. On the homotopy-commutativity of groups and loop spaces. Memoirs of the College of Science, University of Kyoto: Series A, Mathematics, 33:257-269, 1960/61.

[35] Rainer M Vogt. Convenient categories of topological spaces for homotopy theory. Archiv der Mathematik, 22:545-555, 1971.

[36] Rainer M Vogt. Homotopy limits and colimits. Mathematische Zeitschrift, 134:11-52, 1973.

[37] Volkmar Welker, Günter M Ziegler, and Rade T Živaljević. Homotopy colimits - comparison lemmas for combinatorial applications. Journal für die reine und angewandte Mathematik, 509:117-149, 1999.

[38] Günter M Ziegler and Rade T Živaljević. Homotopy types of subspace arrangements via diagrams of spaces. Mathematische Annalen, 295:527-548, 1993.

Department of Mathematics and Mechanics, Moscow State University, 119899 Moscow, Russia

E-mail address: tpanov@mech.math.msu.su

Department of Mathematics, University of Manchester, Manchester M13 9PL, England

E-mail address: nige@ma.man.ac.uk

Fachbereich Mathematik/Informatik, Universität Osnabrück, D-49069 Osnabrück, Germany

E-mail address: rainer@mathematik.uni-osnabrueck.de 